\title{
AND/OR Graph Representation of Assembly Plans
}

\author{
Luiz S. Homem de Mello and Arthur C. Sanderson
}

\section{CMU-RI-TR-86-8}
Department of Electrical and Computer Engincering The Robotics Institute Carnegie-Mellon University Pittsburgh, Pennsylvania 15213

April 1986

\section{Copyright (c) 1986 Carnegic-Mellon University}

This research is supported in part by Conselho Nacional de Desenvolvimento Cientifico e Tecnológico, Brazil, and by the Robotics Institute of Carnegie-Mellon University. 


\section{Table of Contents}

1. Introduction

2. Scheduling and Planning

3. Planning for Robotic Assembly

4. ANI)/OR Graph Representation of Assembly Plans

5. A Simple Example

6. Finding the Best Plan as an AND/OR Graph Scarch

7. Opportunistic Scheduling Using the AND/OR Graph Representation

8. Conclusion 


$$
\text { - }
$$




\section{List of Figures}

Figure 1: $\wedge$ simple product $\quad 4$

Figure 2: Possible sequences of operations to assemble the product of figure 1

Figure 3: Precedence diagrams: (I) combines $\Lambda-B-C$ and $B-A-C$; (2) combines $C-B-\Lambda \quad 6$ and B-A-C; (3) Combines B-A-C and B-C-A

Figure 4: $\Lambda N D / O R$ graph for the product of figure 1

ligure 5: Solution tree corresponding to sequence 4 (C-B-A) in fig. 2

Figure 6: Solution tree corresponding to sequence 8 (E-C-A) in fig. 2

Figure 7: Solution trec corresponding to sequence 3 (B-C-A) in fig. 2

Figure 8: Solution trec corresponding to sequences $6(D-C-\Lambda)$ and $7(C-D-\Lambda)$ in fig. $2 \quad 10$

Figure 9: Solution trec corresponding to sequences $9(\mathrm{E}-\mathrm{A}-\mathrm{C})$ and $10(\mathrm{~A}-\mathrm{E}-\mathrm{C})$ in fig. 2

Figure 10: Solution tree corresponding to sequence 2 (B-A-C) in fig. 2

Figure 11: Solution trec corresponding to sequence 5 (D-A-C) in fig. $2 \quad 12$

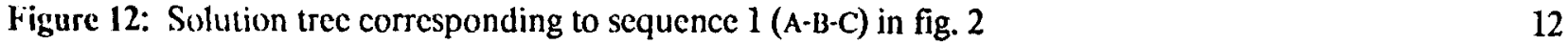

Figure 13: Robotic workstation 15 


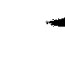




\section{List of Tables}

Table 1: Assignment of wejghts to hyperares

Table 2: Number of operations needed to assemble the product of fig. 1 for all the sequences in which the parts may be acquired, and for the three schemes of plan representation. 


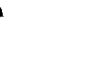




\begin{abstract}
This paper presents a compact representation of all possible assembly plans of a given product using AND/OR graphs. Such a representation forms the basis for efficient planning algorithms which cnable an increase in assembly system flexibility by allowing an intelligent robot to pick a course of action according to instantaneous conditions. 'Two applications are discussed: the selection of the best assembly plan (off-line planning), and opportunistic scheduling (on-line planning). An example of an assembly with four parts illustrates the use of the AND/OR graph representation to find the best assembly plan based on weighing of operations according to complexity of manipulation and stability of subassemblies. In practice, a generic search algorithm, such as the $A O^{*}$ may be used to find this plan. The scheduling efficiency using this representation is compared to fixed sequence and precedence graph representations. The AND/OR graph consistently reduces the average number of operations.
\end{abstract}





\section{Introduction}

Robotic assembly often requires reprogramming or reconfiguration in order to handle a variety of designs in the same system. The design and implementation of such flexible systems is difficult, and automated planning techniques may provide major advantages. Such task planning for robotic asscmbly is critically dependent on the task representation; a new approach to task representation using AND/OR graphs is described in this paper.

Flexibility in robotic workcells provides a number of advantages. Flexible robotic workcells may be reconfigured to handle a wide range of styles and products. Further flexibility can be achieved if those workcells are able to assemble the same product in different ways. In order to accomodate the assembling of several different products in the same shop, it is necessary to schedule the available machines to each job. Since different machines may have different capabilities, the assembly procedure may vary depending on what machine is scheduled to do the job. Also, the same product may be assembled in different shops that may have different machinery. Another advantage is an improvement in the ability to recover from errors and other unexpected cffects that cause the execution of a task to deviate from the preplanned course of actions. When deviations occur, it is preferred that the task execution continue, as cfficiently as possible, from the unpredicted state towards the goal. Many deviations of the desired course of actions are not necessarily crror conditions, but are due to the many random factors that affect the whole manufacturing process, and flexible shops should be able to cope with those factors autonomously.

Even with flexibility of the mechanical hardware, current robotic assembly systems are not able to follow many different courses of actions within a given task. A principal reason for this limitation is the inadequate data structure for the representation of task plans. Ordered lists of actions, that have been used in early robot systems, which were developed outside the manufacturing context, do not permit flexibility in task execution. Triangle tables [4] have been used for the representation of plans, and they improve the capability to recover from errors, but only within one fixed sequence. A more significant improvement was the use of precedence diagrams [5] for the representation of plans, but that technique has limitations also, and in most cases allows only a small amount of flexibility.

This paper presents a compact representation for the set of all possible assembly plans of a given product. Such a representation enables an increase in assembly flexibility by allowing an intelligent robot to pick the more convenient course of actions, according to the instantaneous conditions at the shop. In sections 2 and 3 , the necessary background is established. Section 4 shows the representation, and section 5 presents its use for the assembly of a simple product. Two applications are discussed: section 6 shows how the selection of the best assembly plan can be implemented as a graph search, and section 7 shows the use of the representation in opportunistic scheduling. Section 8 summarizes the contribution of the paper and points to further research. 


\section{Scheduling and Planning}

$\Lambda$ sscmbly of one product requires selection of a sequence of operations and assignment of times and resources for each operation. The problem is usually divided into two parts: planning, or process routing, which is the selection of a sequence of operations, and scheduling, which is the assignment of times and resources.

Scheduling problems, including job-shop scheduling, project scheduling, and assembly-line balancing, have been intensively investigated in Management Sciences and Operations Rescarch [1]. Mathematical programming techniques have most often been used to solve those problems. More recently, the scheduling problem has been studied using contraint-directed reasoning [6].

Planning has been an important rescarch issue in artificial intelligence. BUILD [2] and STRIPS [3, 4] are two early cxamples. Both systems aim to generate plans that enable robots to perform certain tasks. Typically, the tasks consist of achieving a state that satisfies some goal condition from a current state of the world (i.c., the robot environment), and the plans consist of ordered sequences of actions that will transform the initial state into a goal state.

The representation of plans are commonly based on ordered lists of preprogrammed primitive actions. There are some extensions to that representation scheme that enable the robot to take advantage of the work already done in planning, in case unexpected events happen during the execution of a plan. STRIPS, for example, uses a tabular form, called a triangle table, to store a plan. BUILD associates to each primitive action a REASON list (subgoals) as well as a description of the states of the world before and after the action is executed. More recent systems, such as NOAH [9], represent plans as partially ordered sequences of actions with respect to time.

A major emphasis of research work on planning has been on the scarch aspect of the problem, especially control schemes for the search. Priority has been given to develop efficient, powerful and general purpose procedures that can find at least one plan in a wide variety of situations rather than procedures that eventually find the most efficient plan in a more restricted type of situation. In applications where plans are cxecuted one time only, inefficiencies in the plan do not cause any major harm. Also, if plans are generated on line, high speed in plan generation is often preferable to optimal plans.

Search for the most efficient plan requires a criterion to decide whether one plan is better than another. This decision, however, usually requires information available at execution time only and producing the plan in real time may degrade the robot operation, or even be unfeasible, due to the long computing time it usually takes to generate a plan. The choice between planning ahead of time (off line) and planning in real time (on line) is difficult; the former may lead to inefficient plans, whereas the latter may cause a degradation in the robot operation. 


\section{Planning for Robotic Assembly}

To achieve the desired high levels of productivity, the assembly plans must be efficient and keep wasted time and resources to a minimum. Should inefficiencies in the assembly plan of one product be multiplied by the size of the lot, which in common robotic assembly applications ranges from 1,000 to 100,000 units, the resulting total waste may reduce drastically the productivity and may jeopardize the whole process. Conditions at the shop, however, change with time (for example, parts may come in random order), and, usually, there is no single plan that is efficient in every possible situation.

Fox and Kempf [5] address the necd to act opportunistically, as opposed to always follow a preprogrammed fixed order of operations. They suggest that plans gencrated off-line to be given to the robot be a set of operations with minimal ordering constraints. Such a plan was represented by a precedence diagram and would actually encompass several possible sequences of operations that would perform the task of assembling a given product. In real time, depending on the conditions at the shop, the intelligent robot would pick the most appropriate scquence. Using Fox and Kempf notation, the selection of one sequence, and the assignment of operations to specific machines is what is commonly referred to as the scheduling process. Since that selection process involves much less computing time than the planning process, no degradation in the efficiency of the robot operation should occur.

Planning, in this sense, should yield all possible sequences of operations that can be used to assemble a product. That information is the input to the scheduling process, which in real time selects one of those sequences and assigns the machines that will do each operation.

The problem with the precedence diagram formalism, as Fox and Kempf themselves point out, is that for most products no single partial order can encompass every possible assembly sequence. The assembly of the simple product shown in exploded view in figure 1, for example, may be completed by following one of the ten different sequences of operations that are represented graphically in figure 2. It is possible to combine some sequences into one partial order using precedence diagrams. Figure 3 shows three possible ways to combine two of the first four sequences in figure 2; the only restriction is that the insertion of the stick cannot be the last operation. It is possible to combine three of those four sequences into one partial order by using a dummy opcration, but it is not possible to combine the four sequences into one partial order, nor it is possible to combine any of those sequences with the other six sequences in figure 2.

A closer look at the partial ordering representation of plans, in the light of the above assembly example, shows another deficiency of that solution. Two distinct feasible sequences, A-B.C and B-A.C, for example, do not differ simply by the sequence of the operations. Inserting the stick first is not the same operation as inserting it after the receptacle and the cap have been screwed together. The latter operation is probably easier to execute. Similarly, screwing the receptacle and the handle with the stick inside is probably easier to do if the receptacle 




CAP

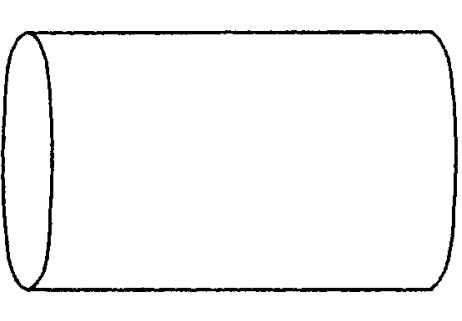

Srick



RECIPTACLE

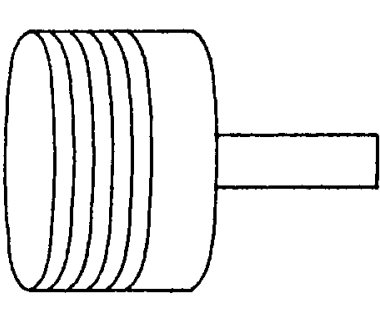

HANDLE

Figure 1: $\Lambda$ simple product

and the cap are screwed, than otherwise. The partial ordering approach, however, does not capture this subtle difference. The next section will describe another approach to the representation of plans that captures this difference, and that can combine all possible assembly sequences.

\section{AND/OR Graph Representation of Assembly Plans}

Planning the assembly of one product made up of several component parts can be seen as path search in the state space of all possible configurations of that set of parts. The initial state is that configuration in which all parts are disconnected from each other, and the goal state is that in which the parts are properly joined to form the desired product. The moves that change one state into another correspond to the assembly operations since they change the relative position of at least one part. There may be many different paths from the initial state to the goal state. Krogh and Sanderson [7] present an overview of task decomposition and operations.

In this context, any set of parts that are joined to form a stable unit is called an assembly. A component part is also an assembly, with a special property. The word subassembly refers to an assembly that is part of another, more complex assembly, and it always carries the subset/set connotation.

Because thcre are many configurations that can be made from the same parts, the branching factor from the initial state to the goal state is greater than the branching factor from the goal state to the initial state. $A$ backward scarch, therefore, will be more efficient than a forward search for the assembly planning problem. The problem of finding how to assemble a given product can be converted to an equivalent problem of finding how the same product can be disassembled. Since assembly operations are not necessarily reversible, the equivalence of the two problems will hold only if the operations used in disassembly are the reverse of a feasible assembly operation regardless of whether these reverse operation themsclves are feasible or not. The expression disassembly operation, therefore, refers to the reverse of a feasible assembly opcration. 


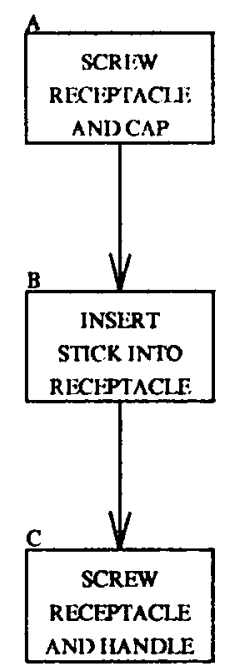

(A-B-C)

(1)

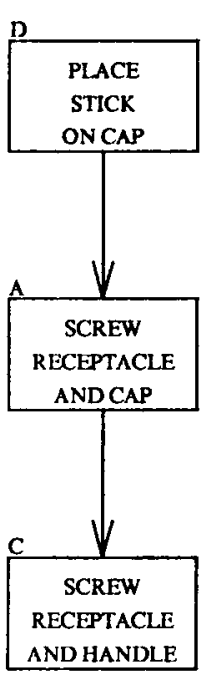

(D-A-C)

(5)
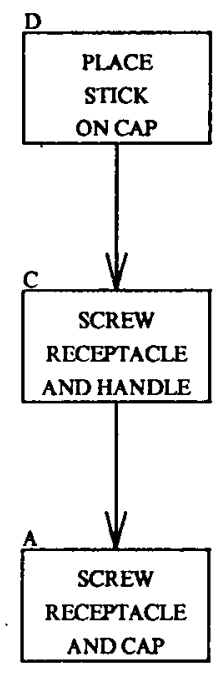

(D-C-A)

(6)



$(\mathrm{B}-\mathrm{A}-\mathrm{C})$

(2)
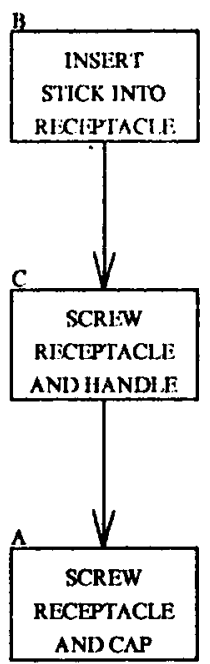

(B-C-A)

(3)


(C-D-A)

(7)
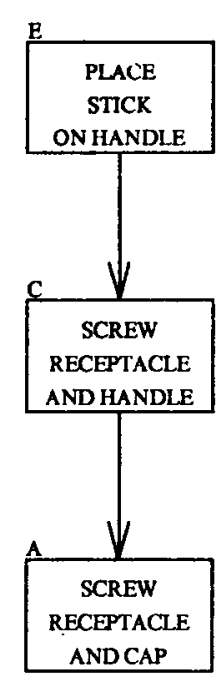

(E-C-A)

(8)
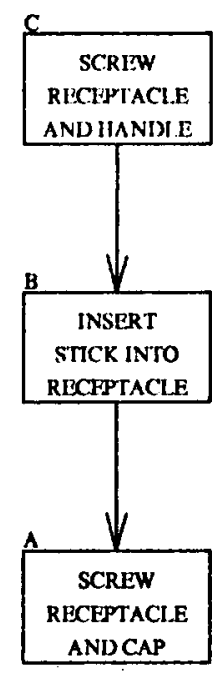

(C-B·A)

(4)
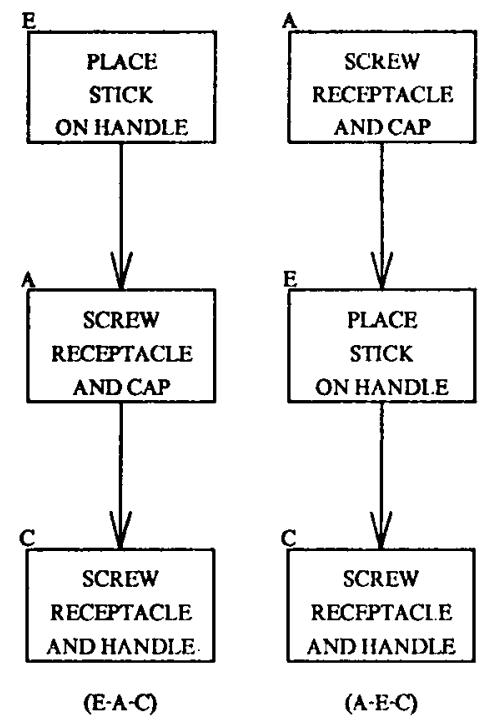

(9)

(10)

Figure 2: Possible sequences of operations to assemble the product of figure 1 




(1)

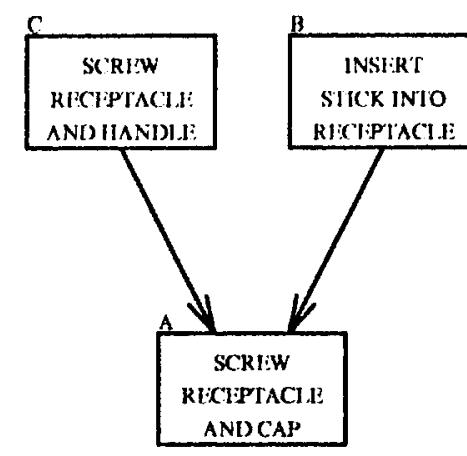

(2)

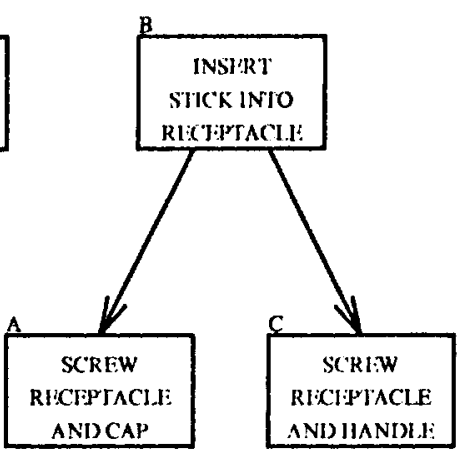

(3)

Figure 3: Precedence diagrams: (I) combines A-B-C and B-A.C; (2) combines C.B.A and B-A.C; (3) combines B-A.C and B.C.A

The backward search suggests a decomposable production system in which the problem of disassembling one product is decomposed into distinct subproblems, each one being to disassemble one subassembly. Each decomposition must correspond to a disassembly operation. If solutions for both subproblems that result from the decomposition are found, then a solution for the original problem can be obtained by combining the solutions to the subproblems and the operation used in the decomposition. For subassemblics that contain one part only, a trivial solution containing no operation always exists. Usually there will not be a unique way to decompose the problem, or to cut the assembly, because there may be several different ways to assemble the same product.

Structures called AND/OR graphs [8], or hypergraphs, are useful in representing decomposable problems and they have been used to represent the disassembly problem. The nodes in such a hypergraph correspond to assemblies; nodes corresponding to assemblies that contain only one part are the terminal nodes. The hyperarcs (or $k$-connectors, $k$ being any integer greater than zero) correspond to the disassembly operations. Each hyperarc that leaves one node corresponds to a disassembly operation applicable to the assembly of that node. and the successor nodes to which the hyperarc points correspond to the resulting subassemblies produced by the disassembly operation. Because for most products the assembly operations usually mate two subassemblies, the hyperarcs in the corresponding AND/OR graph are usually 2-connectors. There are cases, however, of operations that mate more than two subassemblies (e.g, assembling a hinge with two wings and one pin), as well as operations that involve only one subassembly (e.g., drilling a hole in a part). Hyperarcs in AND/OR graphs can represent all those possibilities.

A solution tree from a node $\mathrm{N}$ in an AND/OR graph is a subgraph that may be defined recursively as either $\mathrm{N}$ itself if $\mathrm{N}$ is a terminal node, or $\mathrm{N}$ plus one of its outgoing hyperarcs plus the set of solution trees from each of N's successors through that hyperarc. This definition assumes that the graph contains no cycle as is true in 
the disassembly problem. There may be none, onc. or several solution trecs from a node in an AND/OR graph.

The useful feature of the AND/OR graph representation for the assembly problem is that it encompasses all possible partial orderings of assembly operations. Moreover, cach partial order corresponds to a solution tree from the node corresponding to the final (assembled) product. This feature is demonstrated through the example in the next section.

\section{A Simple Example}

Figure 4 shows the AND/OR graph for the product in figure 1. Fach node in that graph is labeled by a database that correponds to an assembly. In figure 4, the databases are represented by exploded view drawings, whereas in a computational implementation, the databases are relational data structures. 'To facilitate the exposition, both the nodes and the hyperares in figure 4 have identification numbers.

The root node in figure 4 (node 1) is labeled by a database that describes the assembled product. There are four hyperarcs leaving that node. Each of those four hyperarcs corresponds to one way the whole assembly can be disassembled and each one points to two nodes that are labeled by databases that describe the resulting subassemblics. Similarly, the other nodes in the graph have a leaving hyperarc for each possible way in which their corresponding subasscmbly can be disassembled.

Any subassembly that can be made up of the component parts may appear only once in the graph, even when it may be the result of different disassembly operations. The subassembly of node 4 , in figure 4 , for example, may result from two different operations, which correspond to hyperarcs 5 and 10 . Moreover, those two hyperarcs come from two distinct nodes.

Nodes corresponding to component parts (nodes $9,10,11$ and 12) are the terminal or goal nodes since they correspond to disassembling problems for which a (trivial) solution is known. There are eight solution trees from the root node (node 1 ) and they are shown in figures 5 to 12.

One important feature of the solution tree representation shown in figures 5 to 12 is that the distinction between operations becomes apparent because distinct operations correspond to distinct hyperarcs. In other words, two distinct assembly sequences include the same operation only if the two corresponding solution trees include the hyperarc corresponding to that operation. Hyperarc 1, for example, is present in the solution trees in figures 5,6, and 7; therefore, the same assembly operation is part of three distinct sequences. Converscly, the operations SCREW THE RECEPTACLE AND THE CAP in sequences A-B.C, B-A.C, and B.C.A of figure 2 correspond to hyperarcs 1, 5, and 13 in figure 4; therefore, they are three different operations. The sequence diagrams in figure 2 and the precedence diagrams in figure 3 fail to make this distinction. 


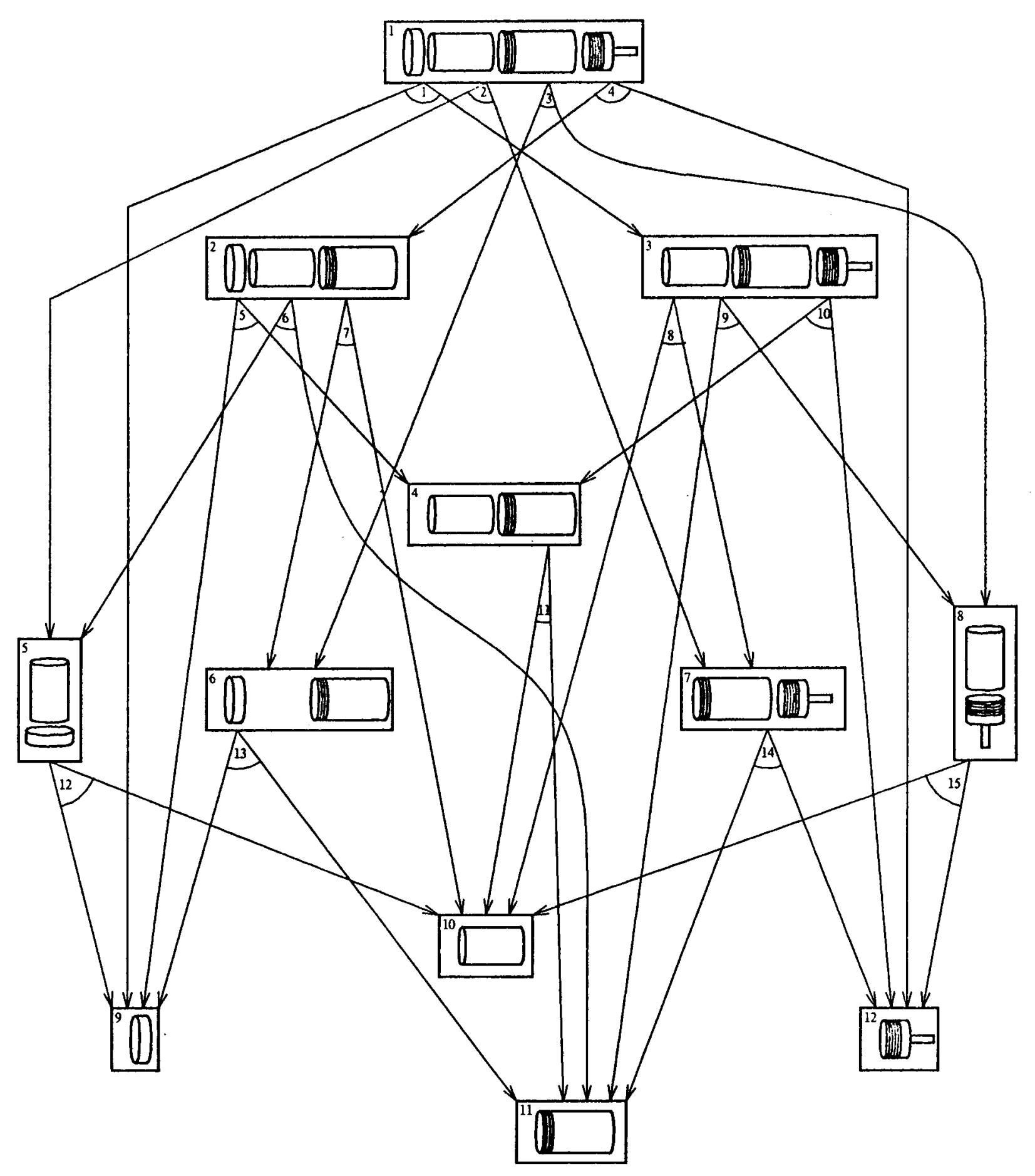

Figure 4: AND/OR graph for the product of figure 1 


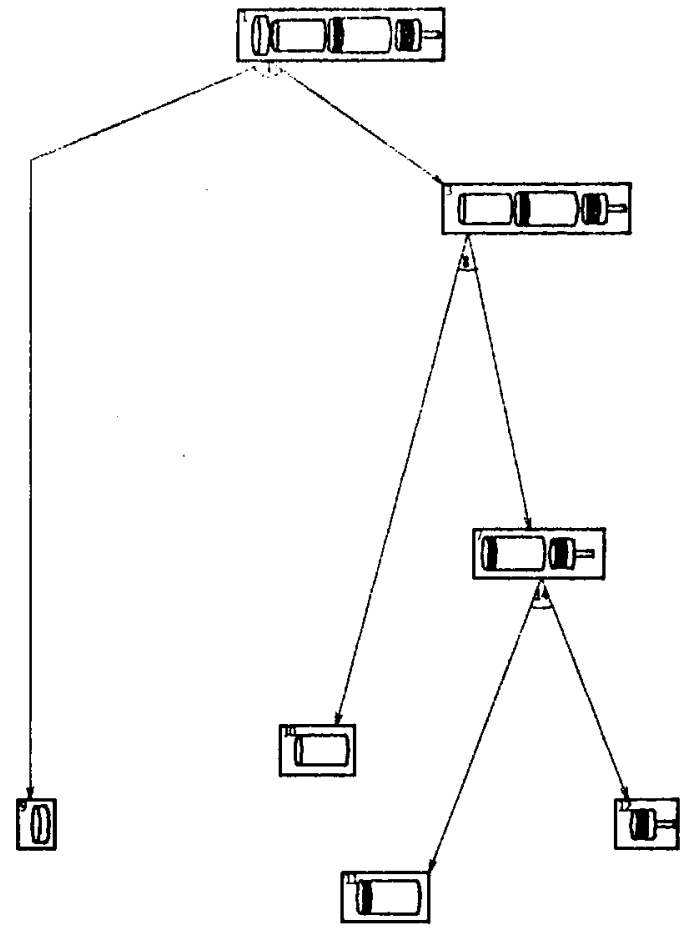

Figure 5: Solution tree corresponding to sequence 4 (C-B-A) in fig. 2

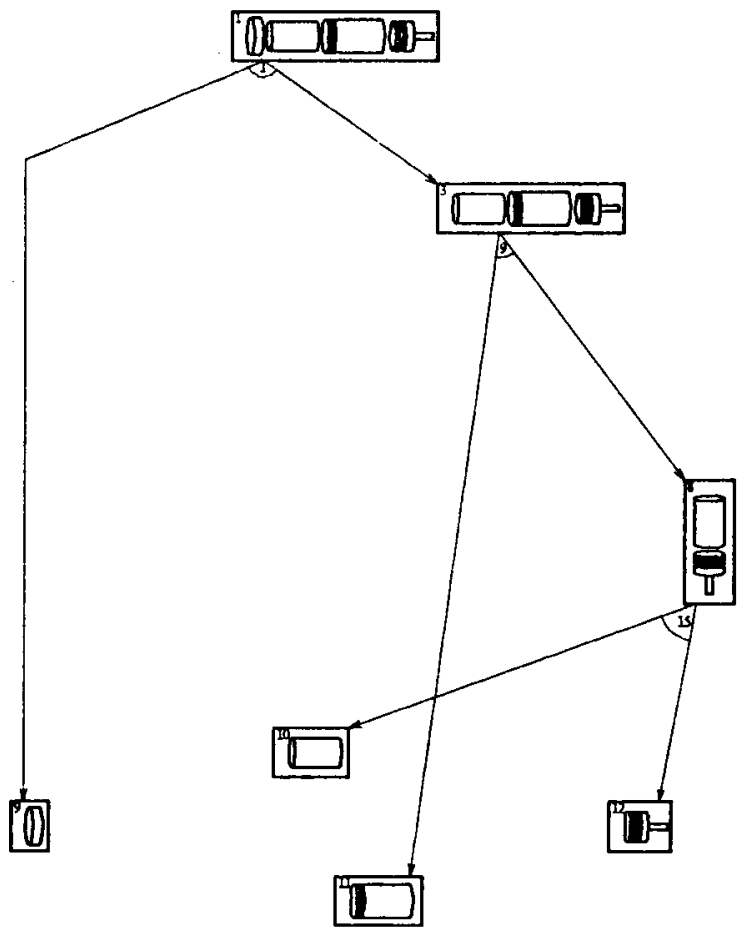

Figure 6: Solution tree corresponding to sequence 8 (E-C-A) in fig. 2 


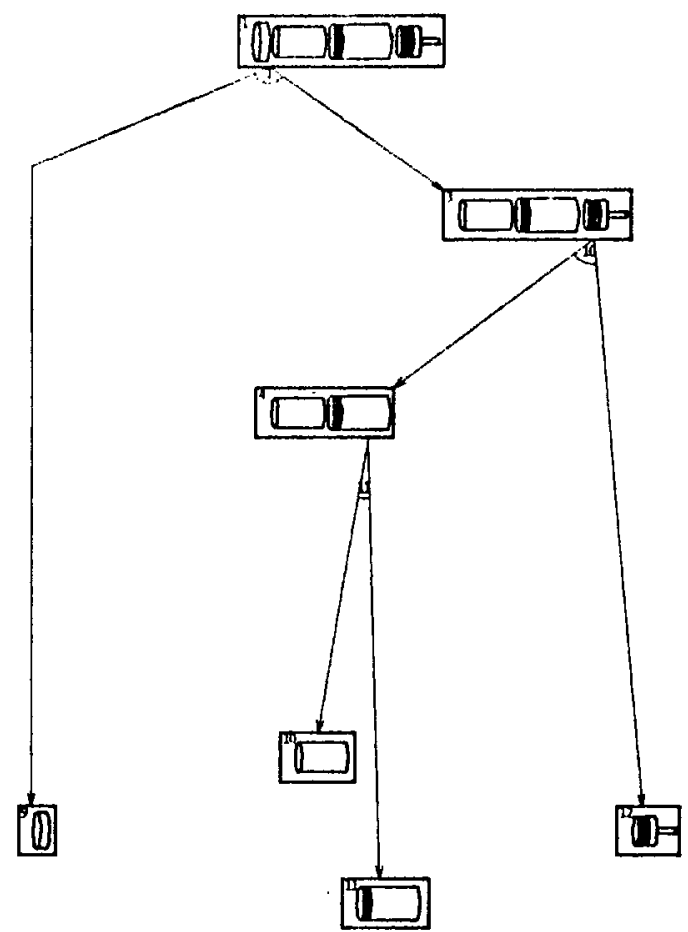

Figure 7: Solution tree corresponding to sequence 3 (B-C-A) in fig. 2

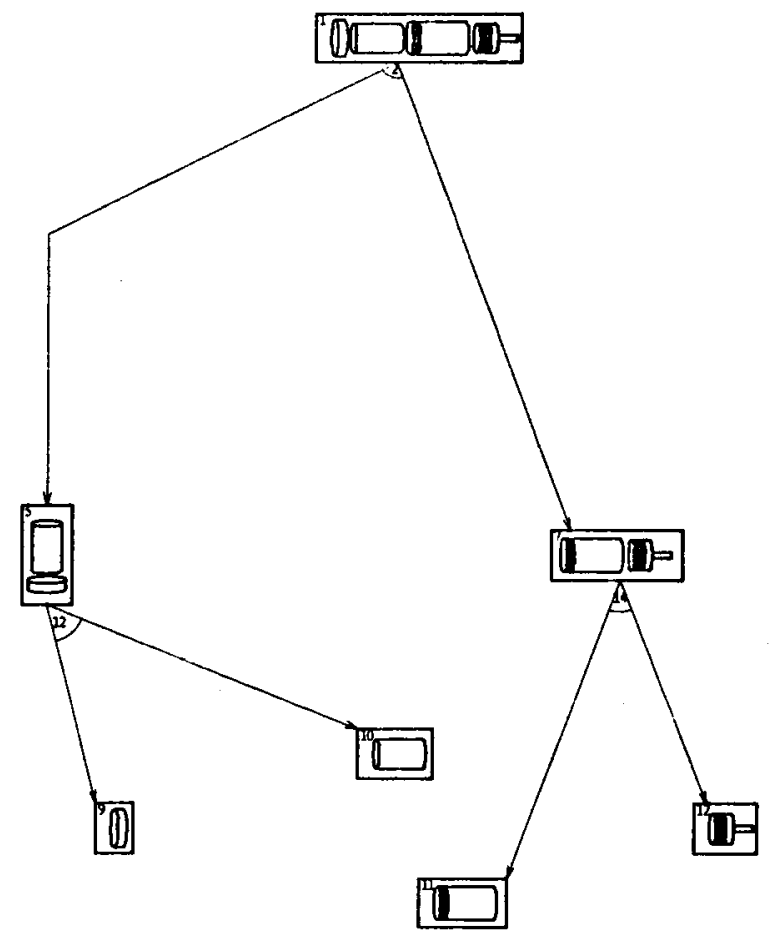

Figure 8: Solution tree corresponding to sequences 6 (D-C-A) and 7 (C-D-A) in fig. 2 


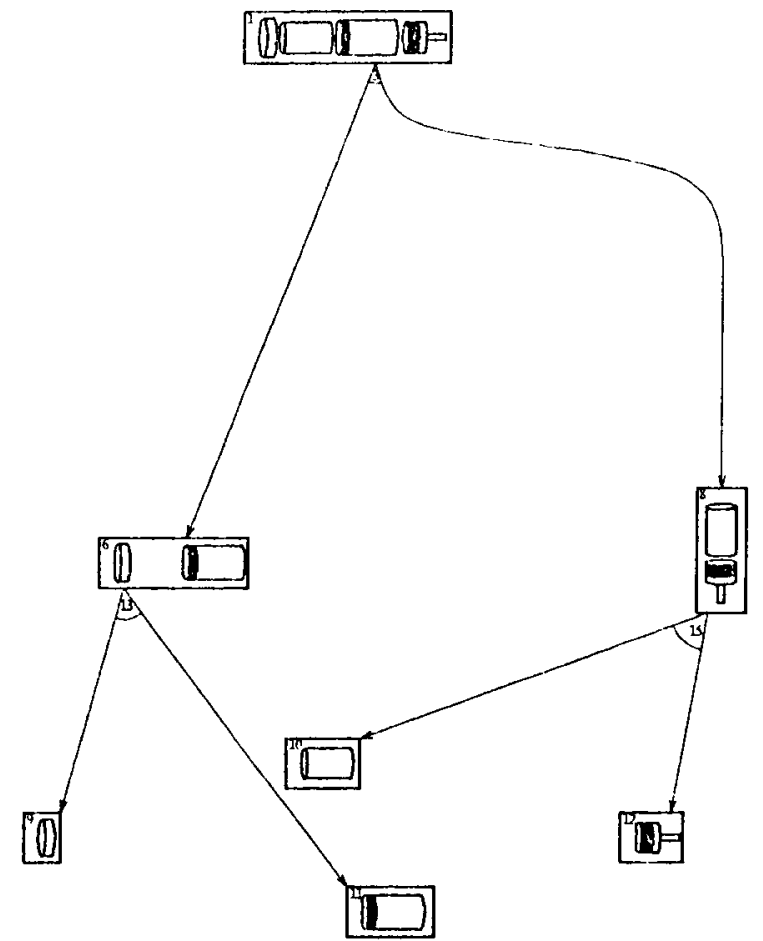

Figure 9: Solution tree corresponding to sequences 9 (E-A-C) and 10 (A-E-C) in fig. 2



Figure 10: Solution tree corresponding to sequence 2 (B-A.C) in fig. 2 


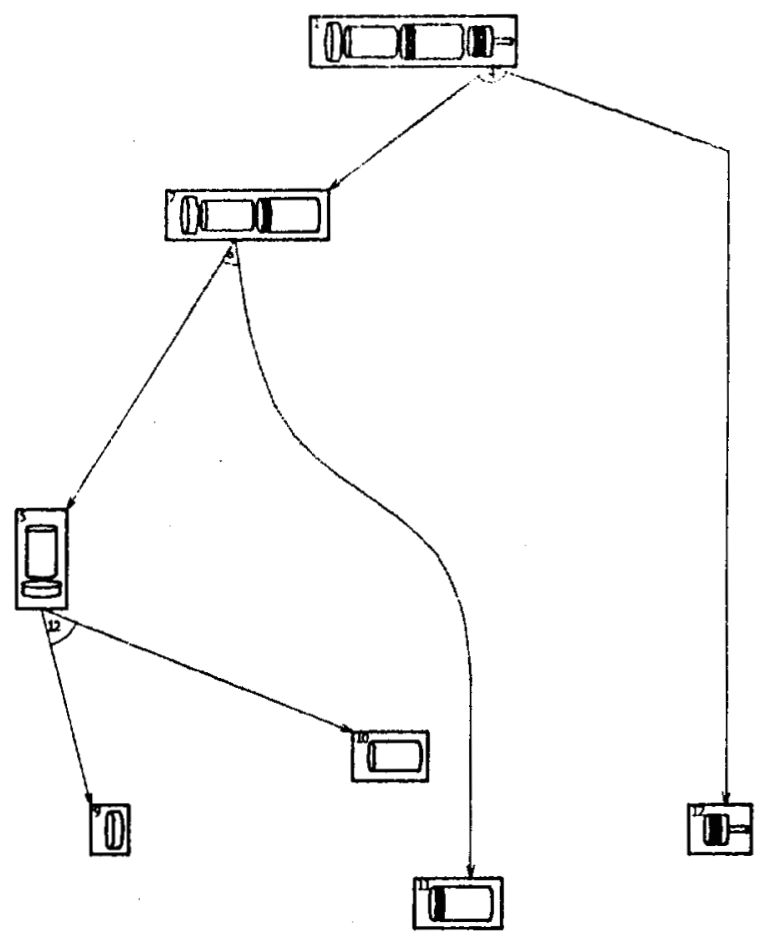

Figure 11: Solution tree corresponding to sequence 5 (D-A-C) in fig. 2

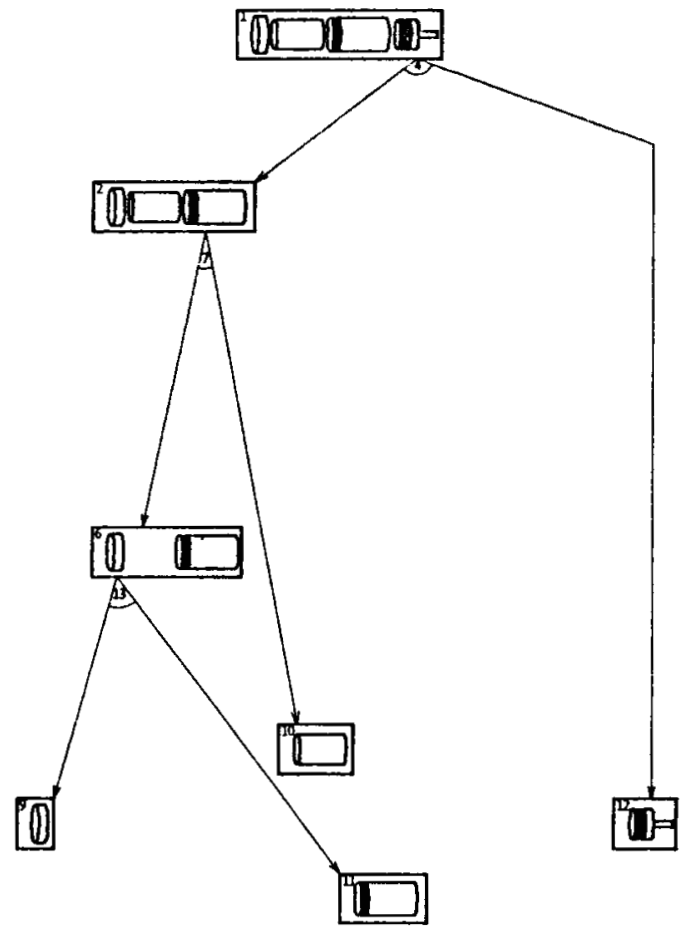

Figure 12: Solution tree corresponding to sequence 1 (A-B-C) in fig. 2 
Each solution tree shown in figures 8 and 9 corresponds to two sequences, but unlike the precedence diagrams of figure 3 , the operations are exactly the same, regardless of the order in which they are executed.

\section{Finding the Best Plan as an AND/OR Graph Search}

To solve problems that require optimization, such as the selection of the best assembly plan, one must be able to traverse the space of all candidate solutions, regardless of the method used to solve the problem. The choice of the representation is critical since it is often difficult to delimit the set of potential solutions in a form which enumerates all the elements.

The AND/OR graph representation encompasses all possible ways to assemble one product, and therefore allows one to explore the space of all possible plans. Since plans correspond to solution trees in the AND/OR graph, the selection of the best plan can be seen as a search problem. Any such search problem requires a criterion to compare plans. One possibility is to assign to the hyperarcs weights proportional to the difficulty of their corresponding operations, and then compute the cost of a solution tree from a node, recursively, as:

- zcro, if the node has no leaving hyperarc; or

- the sum of the weight of the hyperarc leaving the node and the costs of the solution trecs from the successor nodes.

The best plan corresponds to the solution tree that has the minimum cost. The search for the best plan can be conducted using generic algorithms such as the $A O^{*}[8]$.

$\Lambda$ variety of factors might be considered in assigning weights to hyperarcs, including time duration of their corresponding operations, requirements for reorientation of fixturing, cost of resources needed, reliability, as wcll as production priorities and constraints.

For the product in figure 1, the AND/OR graph (figure 4) has 15 hyperarcs, which correspond to 15 different assembly operations. Table 1 shows one possible assignment of weights to hyperarcs. Those weights have been computed by adding two factors. The first factor is the type of assembly operation, with screw operation weighing 4 , insertion 2 and placement 1 , in accord with typical time, fixturing and manipulation requirements. The second factor taken into account is the difficulty of handling the participating subassemblics, and is proportional to their number of degrees of freedom; subassemblies with more degrees of freedom are more unstable, and therefore more difficult to handle.

Using that assignment of weights to hyperarcs, the total cost for the solution trees of figures 5 to 12 can be computed. The solution trees in figures 5 and 12 have the minimum cost of 11 ; the solution trees in figures 7,8 , 9 , and 10 have total cost 13; and the solution trees in figures 6 and 11 have the highest cost of 14 . 
Table 1: Assignment of weights to hyperares

\begin{tabular}{|c|c|c|c|c|c|c|c|c|c|c|c|c|c|c|c|}
\hline \multirow[b]{2}{*}{ factor } & \multicolumn{15}{|c|}{ hypcrarcs } \\
\hline & 1 & 2 & 3 & 4 & 5 & 6 & 7 & 8 & 9 & 10 & 11 & 12 & 13 & 14 & 15 \\
\hline opcration & 4 & 4 & 4 & 4 & 4 & 4 & 2 & 2 & 4 & 4 & 2 & 1 & 4 & 4 & 1 \\
\hline $\begin{array}{l}\text { subassembly } \\
\text { degrees of } \\
\text { frecdom }\end{array}$ & 1 & 4 & 4 & 1 & 2 & 4 & 0 & 0 & 4 & 2 & 0 & 0 & 0 & 0 & 0 \\
\hline total & 5 & 8 & 8 & 5 & 6 & 8 & 2 & 2 & 8 & 6 & 2 & 1 & 4 & 4 & 1 \\
\hline
\end{tabular}

For more complex assemblics, instead of a complete enumeration as done above, search algorithms can be used to reduce computation. For the product in figure 1, a search using AO* will yield one of the solution trees shown in figures 5 or 12, depending on how the partial solutions and tip nodes are ordered for expansion.

\section{Opportunistic Scheduling Using the AND/OR Graph Representation}

To evaluate how the use of AND/OR graph representation for assembly plans affects assembly cfficiency, a comparative analysis among the three representation schemes discussed in this paper has been conducted.

The product in figure 1 , and the robot workstation of figure 13 have been used as examples. The workstation is equipped with two manipulators and the parts are presented in random order. It is assumed that a cap, a stick, a receptacle, and a handle always come together, varying only in their order. It is also assumed that both manipulators are controlled by the same central unit and they both are able to exccute the following actions:

- acquire: fetching, by one of the manipulators, of one part from the part feeder

- buffer: temporararily storing one part into a fixed location within the workstation

- mate: joining two subassemblies which are currently held by the manipulators

- retrieve: fetching, by one of the manipulators, one part known to be in the parts buffer

The efficiency of this assembly station depends on the capacity to handle parts in random order. This requires on-line scheduling of system resources depending on the order of parts arrival. The relative impact of plan representation schemes on assembly efficiency can be compared by the average number of operations needed; a smaller average number of operations corresponds to more efficiency.

The first sequence of figure $2(A-B-C)$ has been used as an example of fixed sequence representation and the 


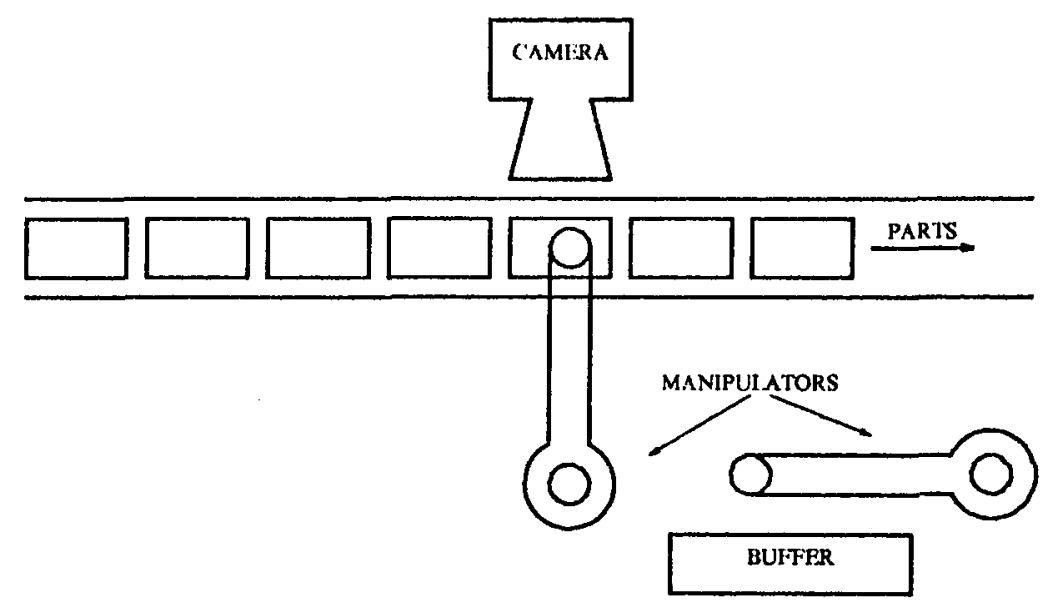

Figure 13: Robotic workstation

first precedence diagram of figure 3 (which combines A-B.C and B-A.C) as an example of precedence graph representation. Similar results will be produced using the other fixed sequences or precedence graphs. The number of operations that would be performed for each one of the 24 possible orderings in which the four parts of the simple product can be acquired is shown in Table 2. At least 7 operations are necessary: four acquisitions and three matings; depending on the order in which the parts are presented, buffering, and therefore retrieving may also be necessary.

When using the fixed sequence representation of plans, extensive buffering is necessary. For example, if the order the parts come is S HR C (stick, handle, receptacle, and cap) both the stick and the handle must be buffered since they are not used in the first operation; adding two bufferings and two retrievings to the four acquisitions and three matings that are always necessary yields 11 operations. The average number of operations for all 24 possible orders is 9.8 .

Using precedence diagrams for the representation of plans avoids some of the buffering and reduces the average number of operations to 9.2. For the sequence SHRC, for example, only the handle must be buffered since the insertion of the stick into the receptacle may be the first operation.

Using the AND/OR graph representation of plans, however, avoids most of the buffering, and yiclds the average of 8 operations. For the same S HRC sequence, for example, no buffering is needed because the robot can follow the sequence of operations corresponding to the solution tree shown in figure 6. 
Table 2: Number of operations needed to assemble the product of fig. I for all the sequences in which the parts may be acquired, and for the three schemes of plan representation.

$$
\mathrm{C}=\text { cap } \mathrm{S}=\text { stick } \mathrm{R}=\text { receptacle } \mathrm{H}=\text { handle }
$$

\begin{tabular}{|c|c|c|c|}
\hline sequence & $\begin{array}{c}\text { first scquence } \\
\text { of figure } 2\end{array}$ & $\begin{array}{l}\text { first precedence diagrams } \\
\text { of figure } 3\end{array}$ & $\begin{array}{c}\text { AND/OR graph } \\
\text { of figure } 4\end{array}$ \\
\hline CSRH & 9 & 9 & 7 \\
\hline C S H R & 11 & 11 & 9 \\
\hline CR SH & 7 & 7 & 7 \\
\hline C R H S & 9 & 9 & 9 \\
\hline CHS R & 11 & 11 & 9 \\
\hline CHRS & 9 & 9 & 9 \\
\hline S CR H & 9 & 9 & 7 \\
\hline SCHR & 11 & 11 & 9 \\
\hline S R C H & 9 & 7 & 7 \\
\hline SR HC & 11 & 9 & 7 \\
\hline SHCR & 11 & 11 & 9 \\
\hline S H R C & 11 & 9 & 7 \\
\hline R C S H & 7 & 7 & 7 \\
\hline RCHS & 9 & 9 & 9 \\
\hline R S C H & 9 & 7 & 7 \\
\hline R S H C & 11 & 9 & 7 \\
\hline R HCS & 9 & 9 & 9 \\
\hline R H S C & 11 & 9 & 7 \\
\hline H C S R & 11 & 11 & 9 \\
\hline HCR S & 9 & 9 & 9 \\
\hline H S C R & 11 & 11 & 9 \\
\hline HS R C & 11 & 9 & 7 \\
\hline H R C S & 9 & 9 & 9 \\
\hline H R S C & 11 & 9 & 7 \\
\hline average & 9.8 & 9.2 & 8 \\
\hline
\end{tabular}




\section{Conclusion}

$\Lambda$ compact representation for the set of all possible assembly plans of a product has been presented, along with its applicitions in the selection of the best assembly plan and in opportunistic scheduling. One important feature of that representation is that it allows one to traverse the space of all possible asscmbly plans, and therefore provides an opportunity to select an optimal schedule and dynamically adapt scheduling to changing conditions. Both the fixed sequence representation and the precedence diagram representation are very limited in this aspect.

A number of issues related to this representation are under investigation. One important issue is the development of algorithms for opportunistic scheduling suitable for real time operation. $\Lambda$ s pointed out in section 7, some buffering could not be avoided, cven with the use of AND/OR graph representation of plans. For complex products, the choice of which part or subassembly to buffer may affect the overall assembly efficiency and criteria for that decision will be necessary. These criteria will certainly depend on evaluation functions, also under investigation, used to select a plan, especially functions that do not possess the recursive property like the one used in section 6 .

An additional important ongoing research issue is the development of a representation of assemblies suitable for the automatic generation of plans. Such automation can be helpful in design of both new products and assembly systems. In designing new products, the designer can quickly assess the difficulty of assembling and eventually modify the design to facilitate the assembly. In designing new assembly systems, the designer can evaluate the performance of a proposed design for a given set of products. 


\section{References}

[1] Bellman, R. et al.

Mathematical Aspects of Scheduling and Applications

Pergamon Press, 1982.

[2] Fahlman, Scott Filiott.

A Planning Systein for Robot Construction 'Tasks.

Artificial Intelligence 5(1):1-49, 1974.

[3] Fikes, Richard E. and Nilsson, Nils J.

SIRIPS: $\Lambda$ New $\Lambda$ pproach to the $\Lambda$ pplication of 'Theorem Proving to Problem Solving.

Artificial Intelligence 2:189-208, 1971.

[4] Fikes, Richard E. et al.

Lcarning and Executing Gencralized Robot Plans.

Artificial Intelligence 3:251-288, 1972.

[5] Fox, B. R. and Kempf, K. G.

Opportunistic Scheduling for Robotics Assembly.

In 1985 IEEE International Conference on Robotics and Automation, pages 880-889. IEEE Computer Society, 1985.

[6] Fox, Mark S.

Constraint-Directed Search: A Case Study of Job-Shop Scheduling.

PhD thesis, Carnegie-Mcllon University, december, 1983.

Also published as technical reports CMU-CS-83-161 and CMU-RI-TR-83-22.

[7] Krogh, Bruce H. and Sanderson, Arthur C.

Modeling and Control of Assembly Tasks and Systems.

Technical Report CMU-RI-'TR-86-1, Robotics Institute - Carncgie-Mellon University, 1985.

[8] Nilsson, Nils J.

Principles of Artificial Intelligence.

Springer-Verlag, 1980.

[9] Sacerdoti, Earl D.

A Structure for Plans and Behavior.

Elsevier North-Holland, 1977. 


\title{
AND / OR GRAPH REPRESENTATION OF ASSEMBLY PLANS
}

\author{
Luiz S. Homem de Mello and Arthur C. Sanderson \\ Department of Electrical and Computer Enginecring \\ and Robotics Institute \\ Carncgic-Mellon University \\ Pittsburgh PA 15213
}

This research is supported in part by Conselho Nacional de Desenvolvimento Cientifico e Tecnológico (Brazil) and by the Robotics Institute of Carnegie-Mellon University. 


\section{Table of Contents}

1. Introduction

2. Scheduling and Planning

3. Planning for Robotic $\Lambda$ sscmbly

4. $\Lambda$ NI)/OR Graph Representation of $\Lambda$ sscmbly Plans

5. ^ Simple Example

6. Finding the Best Plan as an $\Lambda$ NI)/OR Graph Search

7. Opportunistic Scheduling Using the ANI )/OR Graph Representation

8. Conclusion 


\begin{abstract}
This paper presents a compact representation of all possible assembly plans of a given product using AND/OR graphs. Such a representation forms the basis for efficient planning algorithms which enable an increase in assembly system flexibility by allowing an intelligent robot to pick a course of action according to instantancous conditions. Two applications are discussed: the selection of the best asscmbly plan (off-line planning), and opportunistic scheduling (on-line planning). An example of an assembly with four parts illustrates the use of the AND/OR graph representation to find the best assembly plan based on weighing of operations according to complexity of manipulation and stability of subasscmblies. In practice, a generic search algorithm, such as the $\mathrm{AO}^{*}$ may be used to find this plan. The scheduling efficiency using this representation is compared to fixed sequence and precedence graph representations. The AND/OR graph consistently reduces the average number of operations.
\end{abstract}




\section{Introduction}

Robotic assembly often requires reprogramming or reconfiguration in order to handle a variety of designs in the same system. The design and implementation of such flexible systems is difficult, and automated planning techniques may provide major advantages. Such task planning for robotic assembly is critically dependent on the task representation; a new approach to task representation using AND/OR graphs is described in this paper.

Flexibility in robotic workcells provides a number of advantages. Flexible robotic workcclls may be reconfigured to handle a wide range of styles and products. Further flexibility can be achieved if those workcells are able to assemble the same product in different ways. In order to accomodate the assembling of several different products in the same shop, it is necessary to schedule the available machines to cach job. Since different machines may have different capabilities, the assembly procedure may vary depending on what machine is scheduled to do the job. Also, the same product may be assembled in different shops that may have different machinery. Another advantage is an improvement in the ability to recover from crrors and other unexpected effects that cause the execution of a task to deviate from the preplanned course of actions. When deviations occur, it is preferred that the task exccution continue, as efficiently as possible, from the unpredicted state towards the goal. Many deviations of the desired course of actions are not necessarily error conditions, but are due to the many random factors that affect the whole manufacturing process, and flexible shops should be able to cope with those factors autonomously.

Even with flexibility of the mechanical hardware, current robotic assembly systems are not able to follow many different courses of actions within a given task. A principal reason for this limitation is the inadequate data structure for the representation of task plans. Ordered lists of actions, that have been used in early robot systems, which were developed outside the manufacturing context, do not permit flexibility in task execution. Triangle tables [4] have been used for the representation of plans, and they improve the capability to recover from crrors, but only within one fixed sequence. A more significant improvement was the usc of precedence diagrams [5] for the representation of plans, but that technique has limitations also, and in most cases allows only a small amount of flexibility.

This paper presents a compact representation for the set of all possible assembly plans of a given product. Such a representation enables an increase in assembly flexibility by allowing an intelligent robot to pick the more convenient course of actions, according to the instantaneous conditions at the shop. In sections 2 and 3 , the necessary background is established. Section 4 shows the representation, and section 5 presents its use for the assembly of a simple product. Two applications are discussed: section 6 shows how the selection of the best assembly plan can be implemented as a graph search, and section 7 shows the use of the representation in opportunistic scheduling. Section 8 summarizes the contribution of the paper and points to further research. 


\section{Scheduling and Planning}

$\Lambda$ ssembly of one product requires selection of a sequence of operations and assignment of times and resources for each operation. The problem is usually divided into two parts: planning, or process routing, which is the selection of a sequence of operations, and scheduling, which is the assignment of times and resources.

Scheduling problems, including job-shop scheduling, project scheduling, and assembly-line balancing, have been intensively investigated in Management Sciences and Operations Research [1]. Mathematical programming techniques have most often been used to solve those problems. More recently, the scheduling problem has been studied using contraint-directed reasoning [6].

Planning has been an important research issue in artificial intelligence. BUILD [2] and STRIPS [3, 4] are two early examples. Both systems aim to generate plans that enable robots to perform certain tasks. Typically, the tasks consist of achieving a state that satisfies some goal condition from a current state of the world (i.e., the robot environment), and the plans consist of ordered sequences of actions that will transform the initial state into a goal state.

The representation of plans are commonly based on ordered lists of preprogrammed primitive actions. There are some extensions to that representation scheme that enable the robot to take advantage of the work already done in planning, in case unexpected events happen during the execution of a plan. STRIPS, for example, uses a tabular form, called a triangle table, to store a plan. BUILD associates to each primitive action a REASON list (subgoals) as well as a description of the states of the world before and after the action is executed. More recent systems, such as NOAH [9], represent plans as partially ordered sequences of actions with respect to time.

A major emphasis of research work on planning has been on the search aspect of the problem, especially control schemes for the search. Priority has been given to develop efficient, powerful and general purpose procedures that can find at least one plan in a wide variety of situations rather than procedures that eventually find the most efficient plan in a more restricted type of situation. In applications wherc plans are executed one time only, inefficiencies in the plan do not cause any major harm. Also, if plans are generated on line, high speed in plan generation is often preferable to optimal plans.

Search for the most efficient plan requires a criterion to decide whether one plan is better than another. This decision, however, usually requires information available at execution time only and producing the plan in real time may degrade the robot operation, or even be unfeasible, due to the long computing time it usually takes to generate a plan. The choice between planning ahead of time (off line) and planning in real time (on line) is difficult; the former may lead to inefficient plans, whereas the latter may cause a degradation in the robot operation. 


\section{Planning for Robotic Assembly}

To achicve the desired high levels of productivity, the assembly plans must be efficient and keep wasted time and resources to a minimum. Should inefficiencics in the assembly plan of one product be multiplied by the size of the lot, which in common robotic assembly applications ranges from 1,000 to 100,000 units, the resulting total waste may reduce drastically the productivity and may jeopardize the whole process. Conditions at the shop, however, change with time (for example, parts may come in random order), and, usually, there is no single plan that is efficient in cvery possible situation.

Fox and Kempf [5] address the need to act opportunistically, as opposed to always follow a preprogrammed fixed order of operations. They suggest that plans generated off-line to be given to the robot be a set of operations with minimal ordering constraints. Such a plan was represented by a precedence diagram and would actually encompass several possible sequences of operations that would perform the task of assembling a given product. In real time, depending on the conditions at the shop, the intelligent robot would pick the most appropriate sequence. Using Fox and Kempf notation, the selection of one sequence, and the assignment of operations to specific machines is what is commonly referred to as the scheduling process. Since that selection process involves much less computing time than the planning process, no degradation in the efficiency of the robot operation should occur.

Planning, in this sense, should yield all possible scquences of opcrations that can be used to assemble a product. That information is the input to the scheduling process, which in real time selects one of those sequences and assigns the machines that will do each operation.

The problem with the precedence diagram formalism, as Fox and Kempf themselves point out, is that for most products no single partial order can encompass every possible assembly sequence. The assembly of the simple product shown in exploded view in figure 1, for example, may be completed by following one of the ten different sequences of operations that are represented graphically in figure 2. It is possible to combine some sequences into one partial order using precedence diagrams. Figure 3 shows three possible ways to combine two of the first four sequences in figure 2; the only restriction is that the insertion of the stick cannot bc the last operation. It is possible to combine three of those four sequences into one partial order by using a dummy operation, but it is not possible to combine the four sequences into one partial order, nor it is possible to combine any of those sequences with the other six sequences in figure 2.

A closer look at the partial ordering representation of plans, in the light of the above assembly example, shows another deficiency of that solution. Two distinct feasible sequences, A-B.C and B-A.C, for example, do not differ simply by the sequence of the operations. Inserting the stick first is not the same operation as inserting it after the receptacle and the cap have been screwed together. The latter operation is probably easier to execute. Similarly, screwing the receptacle and the handle with the stick inside is probably easier to do if the receptacle 


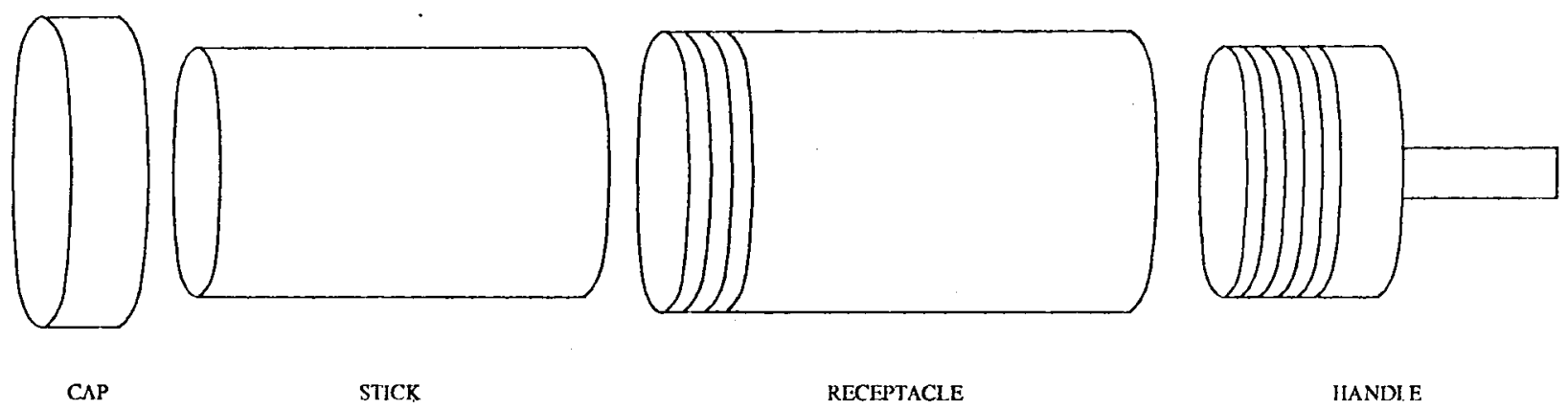

Figure 1: A simple product

and the cap are screwed, than otherwise. The partial ordering approach, however, does not capture this subtle difference. The next section will describe another approach to the representation of plans that captures this difference, and that can combine all possible assembly sequences.

\section{AND/OR Graph Representation of Assembly Plans}

Planning the assembly of one product made up of several component parts can be seen as path search in the state space of all possible configurations of that set of parts. The initial state is that configuration in which all parts are disconnected from each other, and the goal state is that in which the parts are properly joined to form the desired product. The moves that change one state into another correspond to the assembly operations since they change the relative position of at least one part. There may be many different paths from the initial state to the goal statc. Krogh and Sanderson [7] present an overview of task decomposition and operations.

In this context, any set of parts that are joined to form a stable unit is called an assembly. $\Lambda$ component part is also an assembly, with a special property. The word subassembly refers to an assembly that is part of another, more complex assembly, and it always carries the subset/set connotation.

Because there are many configurations that can be made from the same parts, the branching factor from the initial state to the goal state is greater than the branching factor from the goal state to the initial state. A backward search, therefore, will be more efficient than a forward search for the assembly planning problem. The problem of finding how to assemble a given product can be converted to an equivalent problem of finding how the same product can be disassembled. Since assembly operations are not necessarily reversible, the equivalence of the two problems will hold only if the operations used in disassembly are the reverse of a feasible assembly operation regardless of whether these reverse operation themselves are feasible or not. The expression disassembly operation, therefore, refers to the reverse of a feasible assembly operation. 


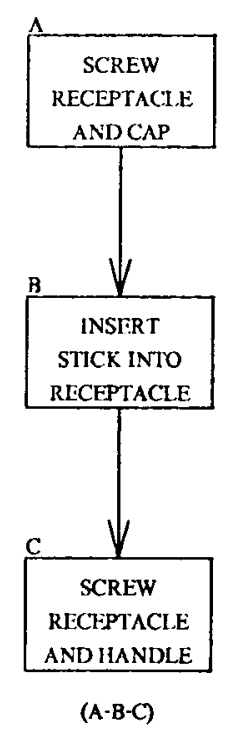

(1)

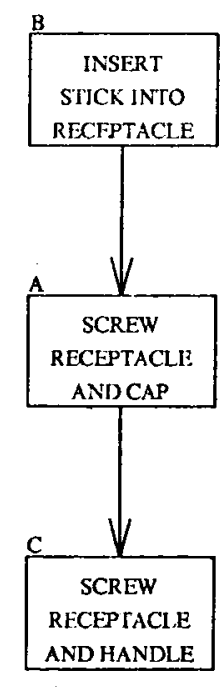

(B-A-C)

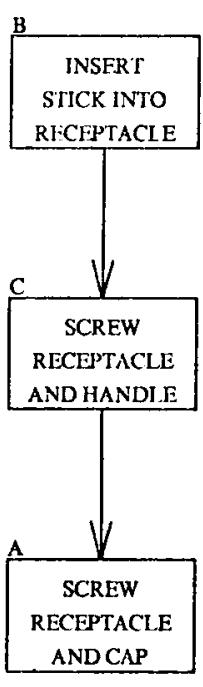

(B-C-A)
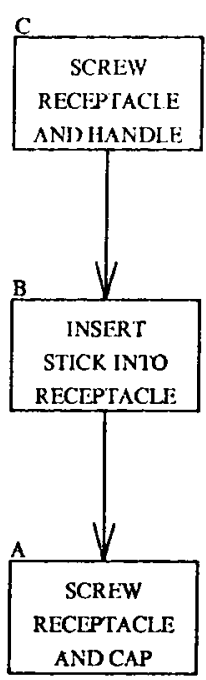

(C-B-A)

(4)
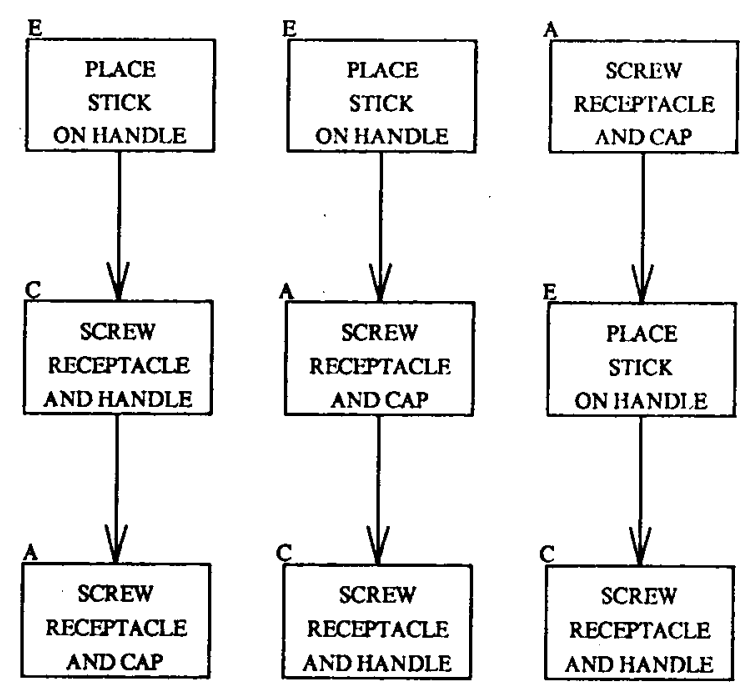

(E-C-A)

(8)

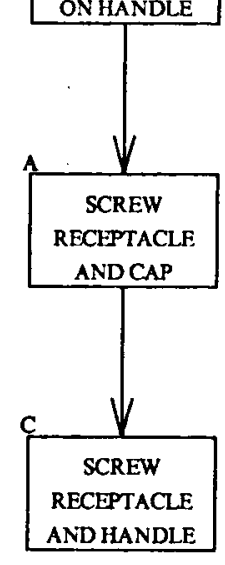

$(\mathrm{E}-\mathrm{A}-\mathrm{C})$
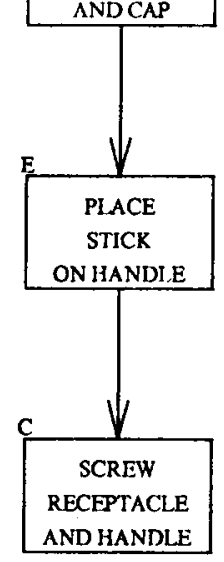

(A-E-C)

(5)

(6)

(7)

(9)

(10)

Figure 2: Possible sequences of operations to assemble the product of figure 1 


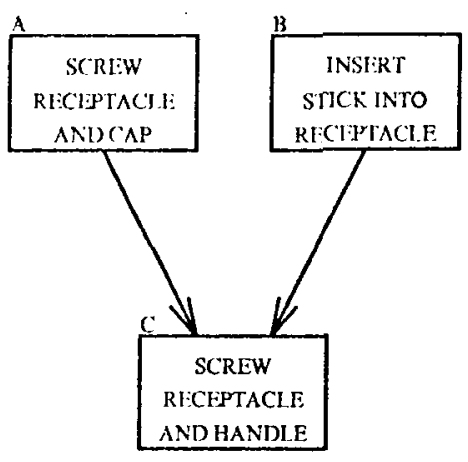

(1)

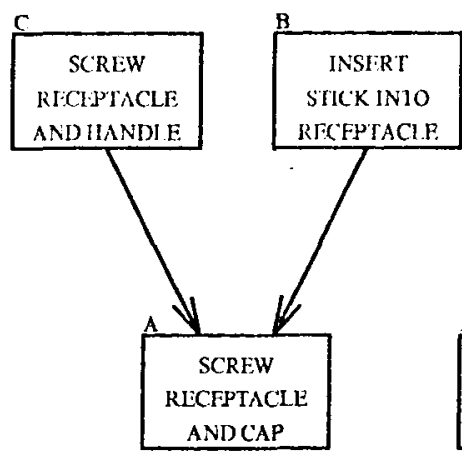

(2)

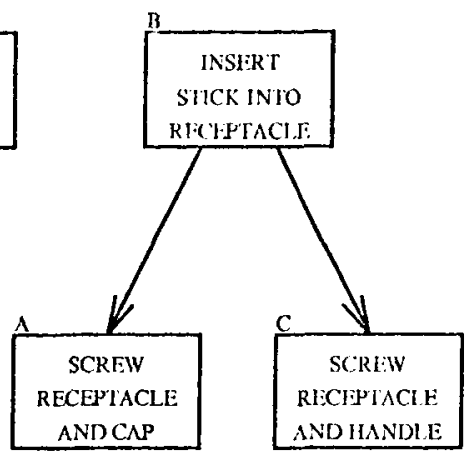

(3)

Figure 3: Precedence diagrams: (I) combines A-B.C and B-A.C; (2) combines C-B-A and B-A.C; (3) combines B-A.C and B-C.A

The backward search suggests a decomposable production system in which the problem of disassembling one product is decomposed into distinct subproblems, each one being to disassemble one subassembly. Each decomposition must correspond to a disassembly operation. If solutions for both subproblems that result from the decomposition are found, then a solution for the original problem can be obtained by combining the solutions to the subproblems and the operation used in the decomposition. For subassemblies that contain one part only, a trivial solution containing no opcration always exists. Usually there will not be a unique way to decompose the problem, or to cut the assembly, because there may be several different ways to assemble the same product.

Structures called AND/OR graphs [8], or hypergraphs, are useful in representing decomposable problems and they have been used to represent the disassembly problem. The nodes in such a hypergraph correspond to assemblics; nodes corresponding to assemblies that contain only one part are the terminal nodes. The hyperarcs (or $k$-connectors, $k$ being any integer greater than zero) correspond to the disassembly operations. Each hyperarc that leaves one node corresponds to a disassembly operation applicable to the assembly of that node, and the successor nodes to which the hyperarc points correspond to the resulting subassemblies produced by the disassembly operation. Because for most products the assembly operations usually mate two subassemblies, the hyperarcs in the corresponding AND/OR graph are usually 2-connectors. There are cases, however, of operations that mate more than two subassemblies (e.g, assembling a hinge with two wings and one pin), as well as operations that involve only one subassembly (e.g., drilling a hole in a part). Hyperarcs in ANO/OR graphs can represent all those possibilities.

A solution tree from a node $\mathrm{N}$ in an AND/OR graph is a subgraph that may be defined recursively as either $\mathrm{N}$ itself if $\mathrm{N}$ is a terminal node, or $\mathrm{N}$ plus one of its outgoing hyperarcs plus the set of solution trees from each of N's successors through that hyperarc. This definition assumes that the graph contains no cycle as is true in 
the disassembly problem. There may be none, one, or several solution trees from a node in an AND/OR graph.

The useful feature of the AND/OR graph representation for the assembly problem is that it encompasscs all possible partial orderings of assembly operations. Morcover, each partial order corresponds to a solution tree from the node corresponding to the final (assembled) product. 'This feature is demonstrated through the example in the next section.

\section{A Simple Example}

Figure 4 shows the AND/OR graph for the product in figure 1. Each node in that graph is labeled by a database that correponds to an assembly. In figure 4, the databases are represented by exploded view drawings, whereas in a computational implementation, the databases are relational data structures. To facilitate the exposition, both the nodes and the hyperarcs in figure 4 have identification numbers.

The root node in figure 4 (node 1 ) is labeled by a database that describes the assembled product. There are four hyperarcs leaving that node. Each of those four hyperarcs corresponds to one way the whole assembly can be disassembled and each one points to two nodes that are labeled by databases that describe the resulting subassemblies. Similarly, the other nodes in the graph have a leaving hyperarc for each possible way in which their corresponding subassembly can be disassembled.

Any subassembly that can be made up of the component parts may appear only once in the graph, even when it may be the result of different disassembly operations. The subassembly of node 4, in figure 4, for example, may result from two different operations, which correspond to hyperarcs 5 and 10 . Moreover, those two hyperarcs come from two distinct nodes.

Nodes corresponding to component parts (nodes 9,10,11 and 12) are the terminal or goal nodes since they correspond to disassembling problems for which a (trivial) solution is known. There are eight solution trees from the root node (node 1) and they are shown in figures 5 to 12.

One important feature of the solution tree representation shown in figures 5 to 12 is that the distinction between operations becomes apparent because distinct operations correspond to distinct hyperarcs. In other words, two distinct assembly sequences include the same operation only if the two corresponding solution trees include the hyperarc corresponding to that operation. Hyperarc 1, for example, is present in the solution trees in figures 5,6, and 7; therefore, the same assembly operation is part of three distinct sequences. Conversely, the operations SCREW THE RECEPTACLE AND THE CAP in sequences A-B-C, B-A-C, and B-C-A of figure 2 correspond to hyperarcs 1,5 , and 13 in figure 4; therefore, they are three different operations. The sequence diagrams in figure 2 and the precedence diagrams in figure 3 fail to make this distinction. 


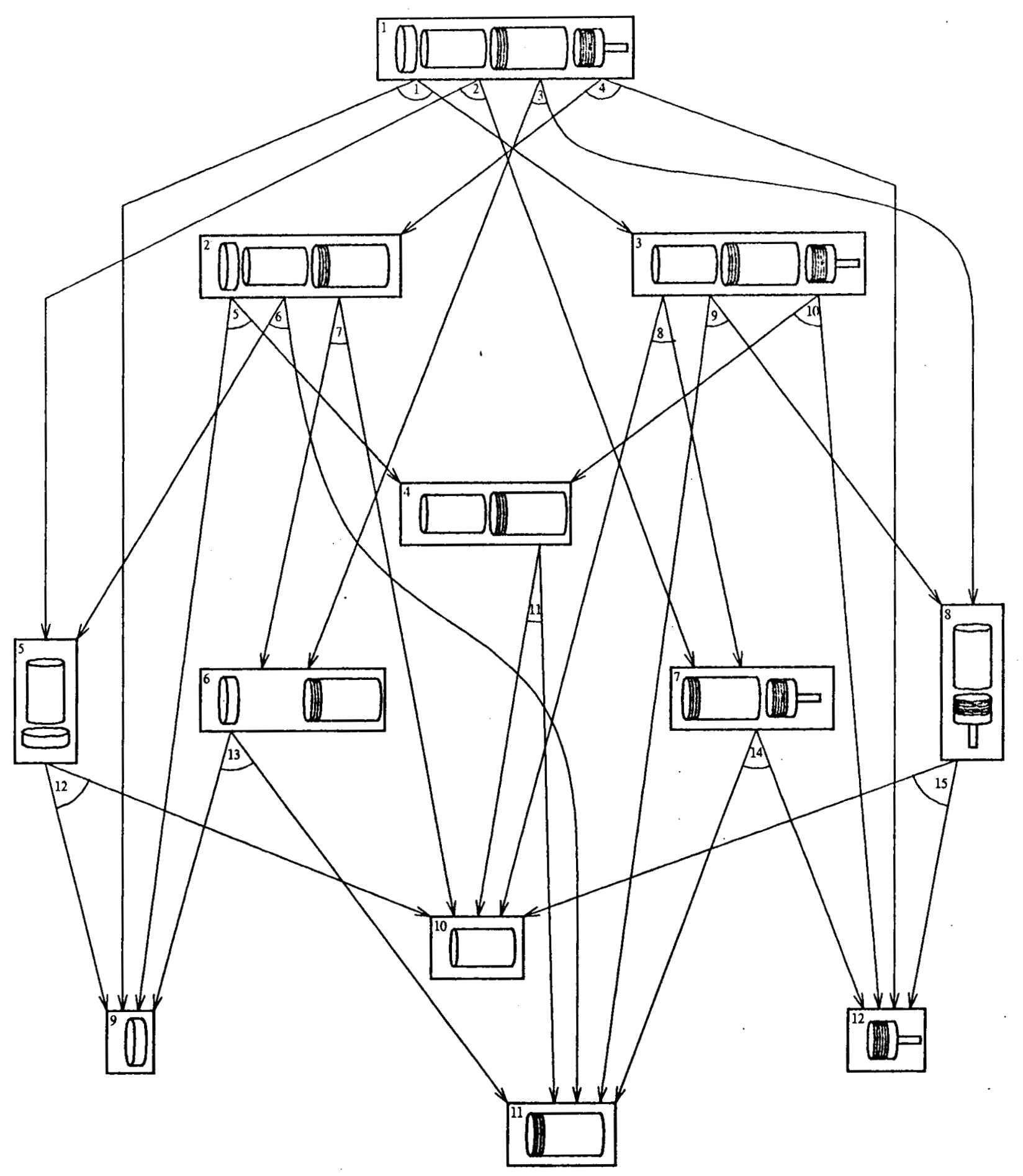

Figure 4: AND-OR graph for the product of figure 1 


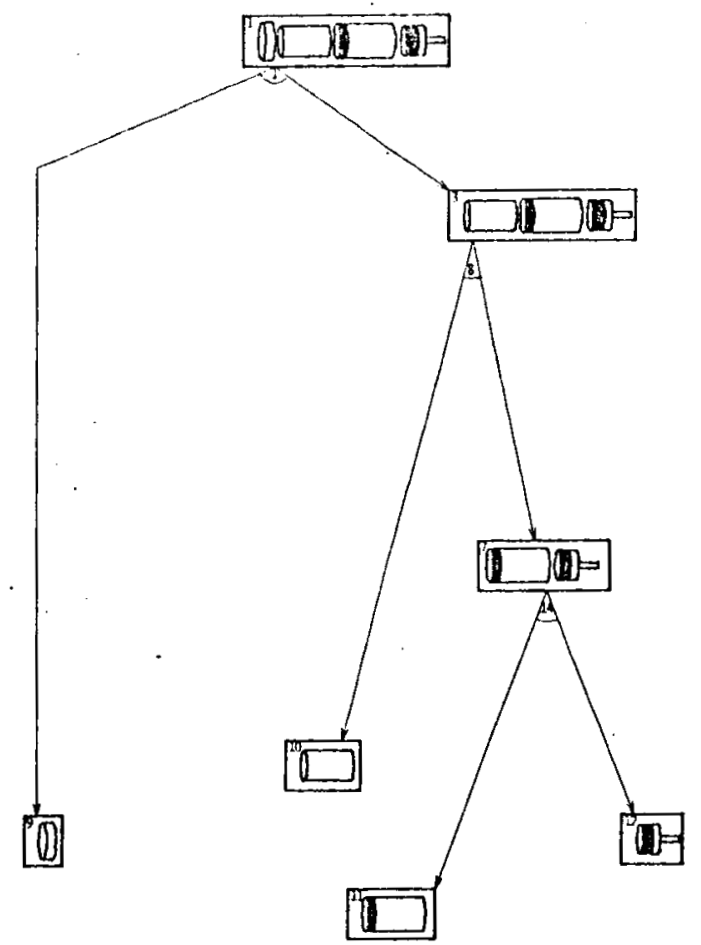

Figure 5: Solution tree corresponding to sequence 4.(C-B-A) in fig. 2

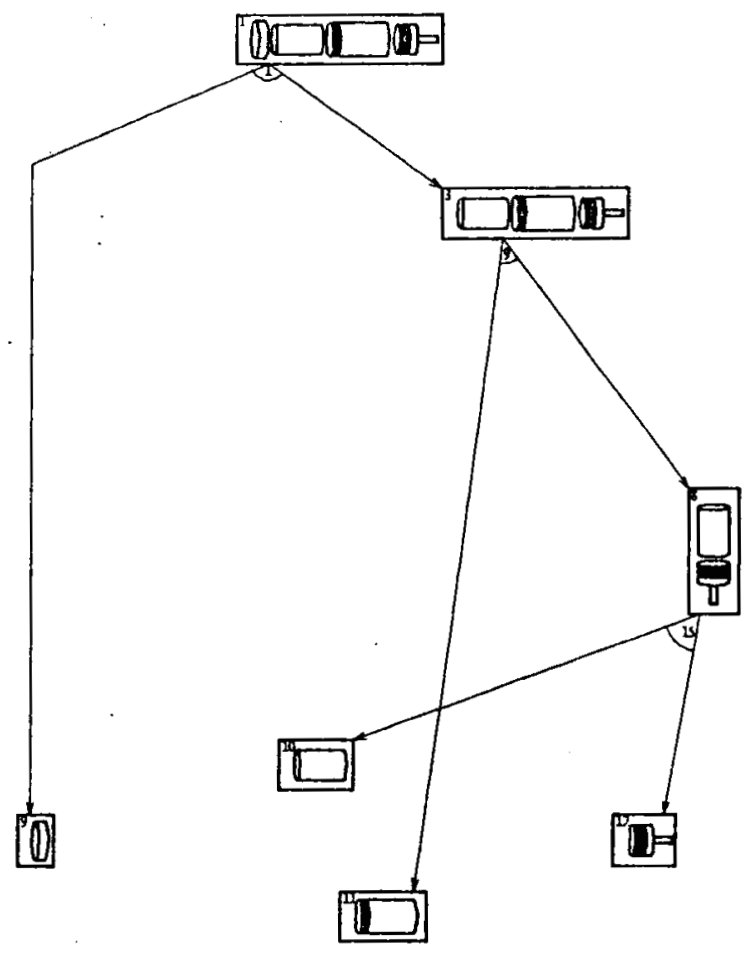

Figure 6: Solution tree corresponding to sequence 8 (E-C-A) in fig. 2 


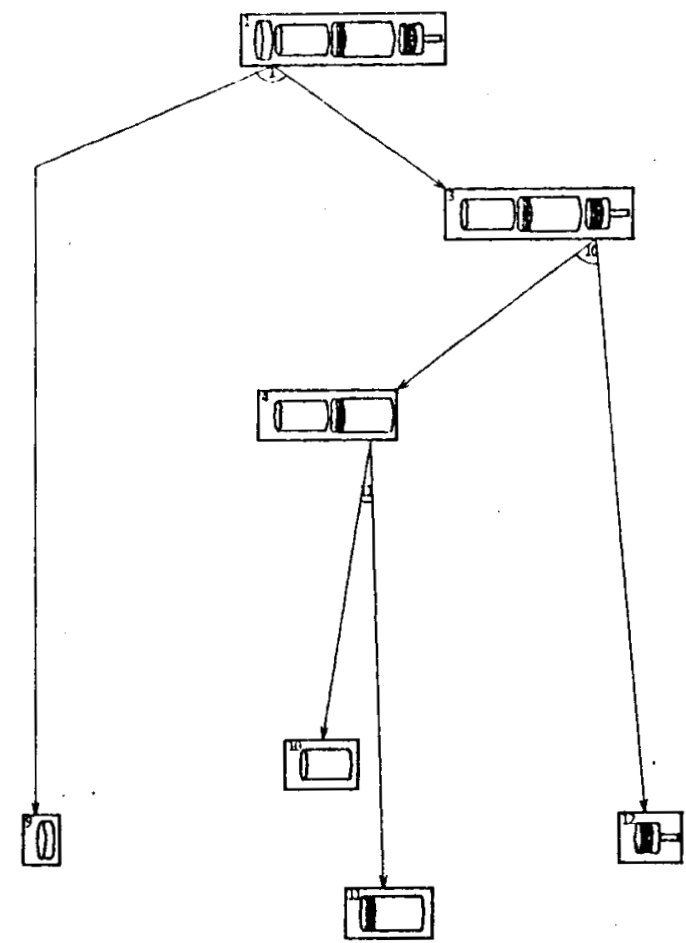

Figure 7: Solution tree corresponding to sequence 3 (B-C-A) in fig. 2

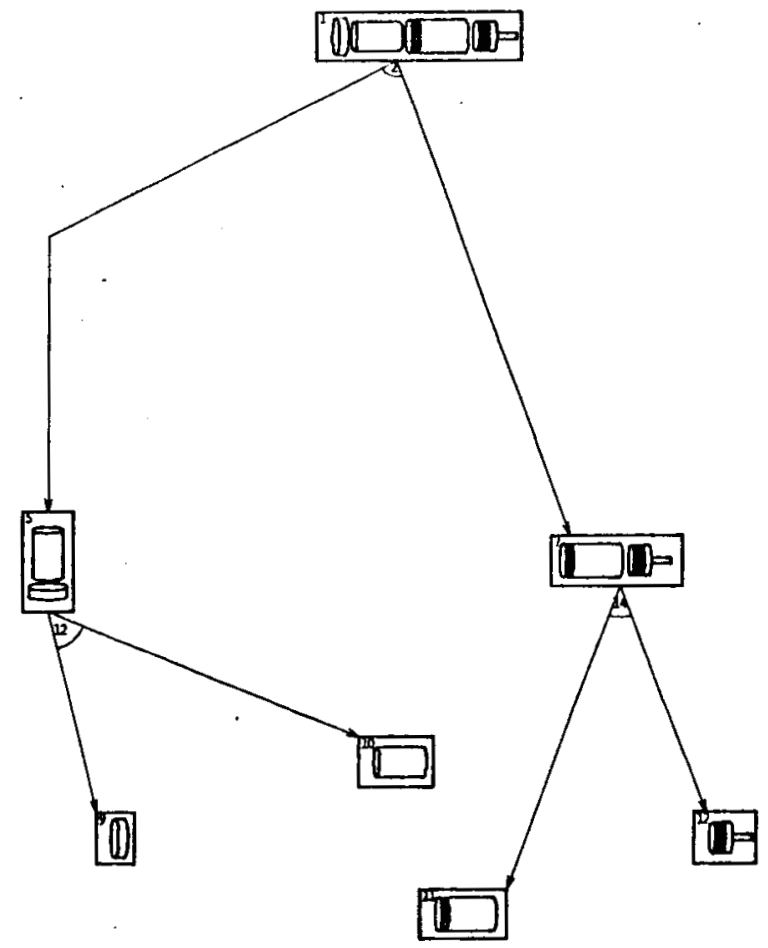

Figure 8: Solution tree corresponding to sequences 6 (D.C.A) and 7 (C.D.A) in fig. 2 


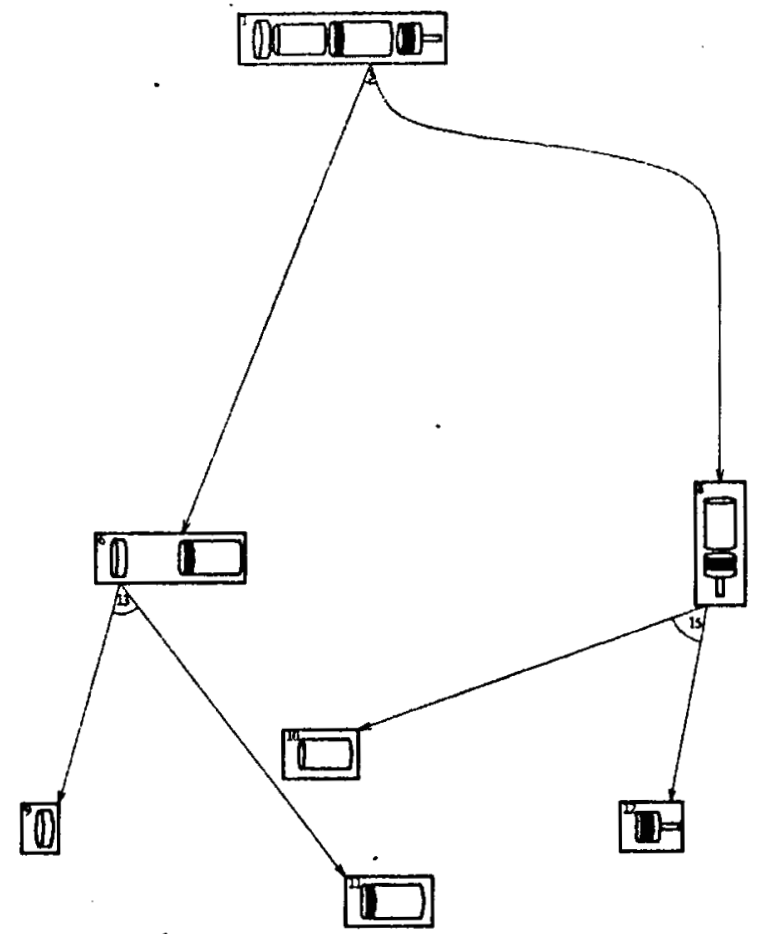

Figure 9: Solution tree corresponding to sequences 9 (E-A-C) and 10 (A-E-C) in fig. 2

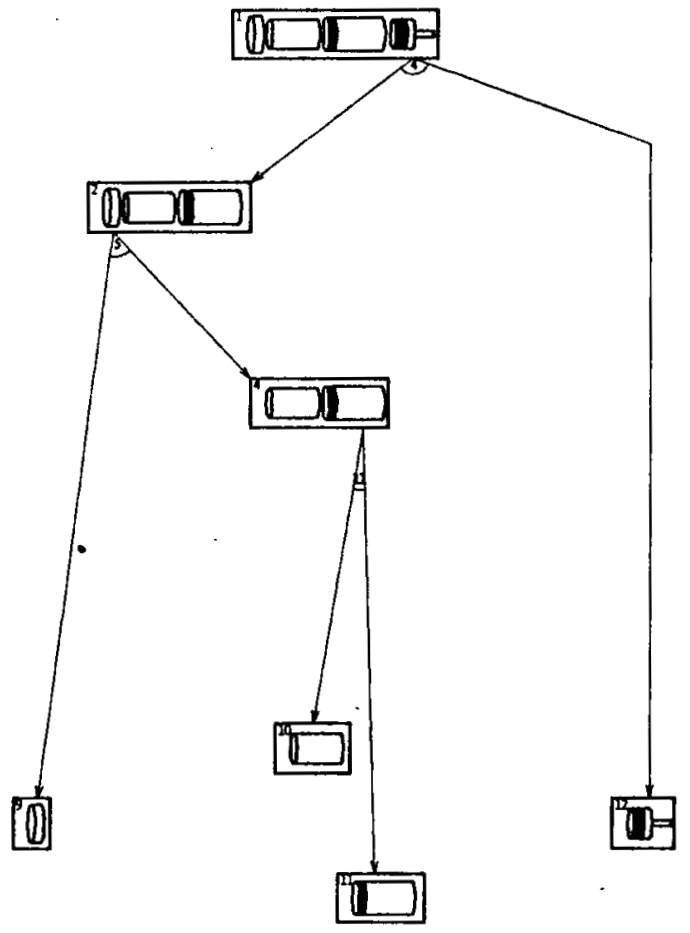

Figure 10: Solution tree corresponding to sequence 2 (B-A-C) in fig. 2 


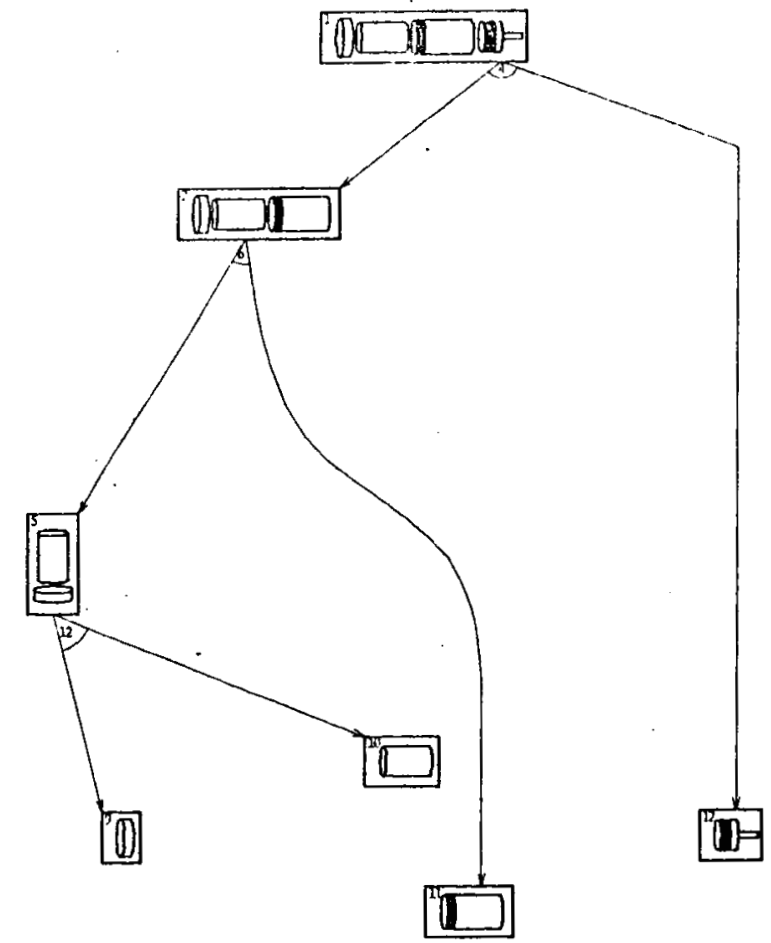

Figure 11: Solution tree corresponding to sequence 5 (D-A-C) in fig. 2

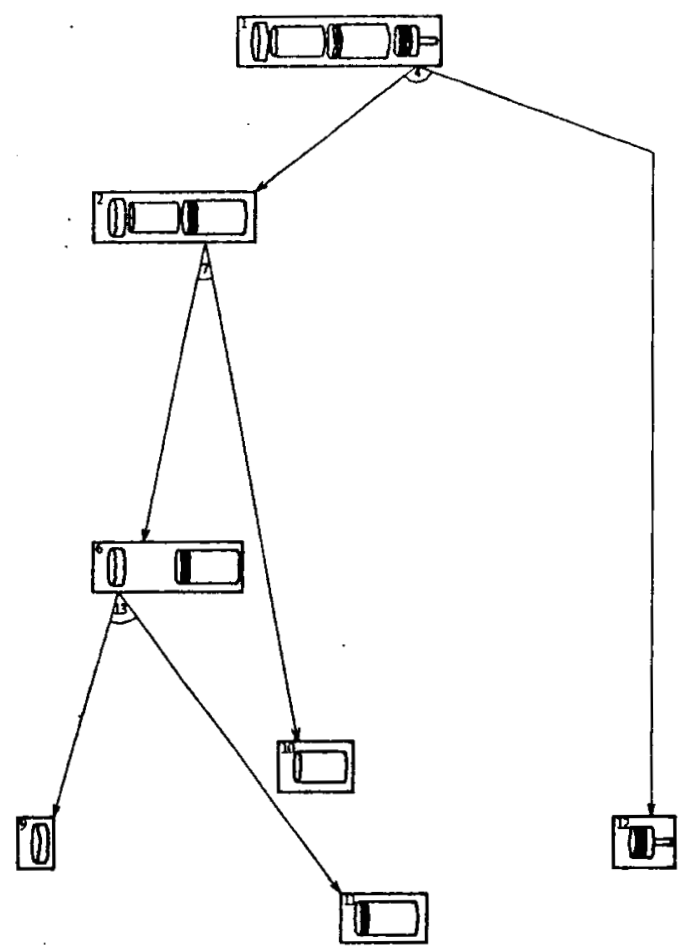

Figure 12: Solution tree corresponding to sequence 1 (A-B-C) in fig. 2 
Fach solution tree shown in figures 8 and 9 corresponds to two sequences, but unlike the precedence diagrams of figure 3 , the operations are exactly the same, regardless of the order in which they are exceuted.

\section{Finding the Best Plan as an AND/OR Graph Search}

To solve problems that requirc optimization, such as the sclection of the best assembly plan, one must be able to traverse the space of all candidate solutions, regardless of the method used to solve the problem. 'The choice of the representation is critical since it is often difficult to delimit the set of potential solutions in a form which enumerates all the elements.

The AND/OR graph representation encompasses all possible ways to assemble one product, and therefore allows one to explore the space of all possible plans. Since plans correspond to solution trees in the AND/OR graph, the selection of the best plan can be seen as a search problem. Any such search problem requires a criterion to compare plans. One possibility is to assign to the hyperarcs weights proportional to the difficulty of their corresponding operations, and then compute the cost of a solution trce from a node, recursively, as:

- zero, if the node has no leaving hyperarc; or

- the sum of the weight of the hyperarc leaving the node and the costs of the solution trees from the successor nodes.

The best plan corresponds to the solution tree that has the minimum cost. The search for the best plan can be conducted using generic algorithms such as the $\mathrm{AO}^{*}[8]$.

A variety of factors might be considered in assigning weights to hyperarcs, including time duration of their corresponding operations, requirements for reorientation of fixturing, cost of resources needed, reliability, as well as production priorities and constraints.

For the product in figure 1, the AND/OR graph (figure 4) has 15 hyperarcs, which correspond to 15 different assembly operations. Table 1 shows one possible assignment of weights to hyperarcs. Those weights have been computed by adding two factors. The first factor is the type of assembly operation, with screw operation weighing 4, insertion 2 and placement 1 ; in accord with typical time, fixturing and manipulation requirements. The sccond factor taken into account is the difficulty of handling the participating subassemblies, and is proportional to their number of degrees of freedom; subassemblies with more degrees of freedom are more unstable, and therefore more difficult to handle.

Using that assignment of weights to hyperarcs, the total cost for the solution trees of figures 5 to 12 can be computed. The solution trees in figures 5 and 12 have the minimum cost of 11 ; the solution trees in figures 7,8 , 9 , and 10 have total cost 13; and the solution trees in figures 6 and 11 have the highest cost of 14 . 
Table 1: Assignment of weights to hyperarcs

\begin{tabular}{ccccccccccccccccc}
\hline & 1 & 2 & 3 & 4 & 5 & 6 & 7 & 8 & 9 & 10 & 11 & 12 & 13 & 14 & 15 \\
\hline factor & 4 & 4 & 4 & 4 & 4 & 4 & 2 & 2 & 4 & 4 & 2 & 1 & 4 & 4 & 1 \\
\hline $\begin{array}{c}\text { operation } \\
\text { subasscmbly } \\
\text { degrees of } \\
\text { freedom }\end{array}$ & 1 & 4 & 4 & 1 & 2 & 4 & 0 & 0 & 4 & 2 & 0 & 0 & 0 & 0 & 0 \\
\hline total & 5 & 8 & 8 & 5 & 6 & 8 & 2 & 2 & 8 & 6 & 2 & 1 & 4 & 4 & 1 \\
\hline
\end{tabular}

For more complex assemblies, instead of a complete cnumeration as done above, search algorithms can be used to reduce computation. For the product in figure 1, a search using $\mathrm{AO}^{*}$ will yield one of the solution trees shown in figures 5 or 12, depending on how the partial solutions and tip nodes are ordered for expansion.

\section{Opportunistic Scheduling Using the AND/OR Graph Representation}

To evaluate how the use of AND-OR graph representation for assembly plans affects assembly cfficiency, a comparative analysis among the three representation schemes discussed in this paper has been conducted.

The product in figure 1 , and the robot workstation of figure 13 have been used as examples. The workstation is equipped with two manipulators and the parts are presented in random order. It is assumed that a cap, a stick, a receptacle, and a handle always come together, varying only in their order. It is also assumed that both manipulators are controlled by the same central unit and they both are able to exccute the following actions:

- acquire: fetching, by one of the manipulators, of one part from the part feeder

- buffer: temporararily storing one part into a fixed location within the workstation

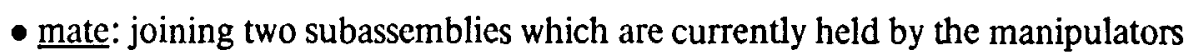

- rctrieve: fetching, by one of the manipulators, one part known to be in the parts buffer

The efficiency of this assembly station depends on the capacity to handle parts in random order. This requires on-line scheduling of system resources depending on the order of parts arrival. The relative impact of plan representation schemes on assembly efficiency can be compared by the average number of operations needed; a smaller average number of operations corresponds to more efficiency.

The first sequence of figure 2 (A-B-C) has been used as an example of fixed sequence representation and the 


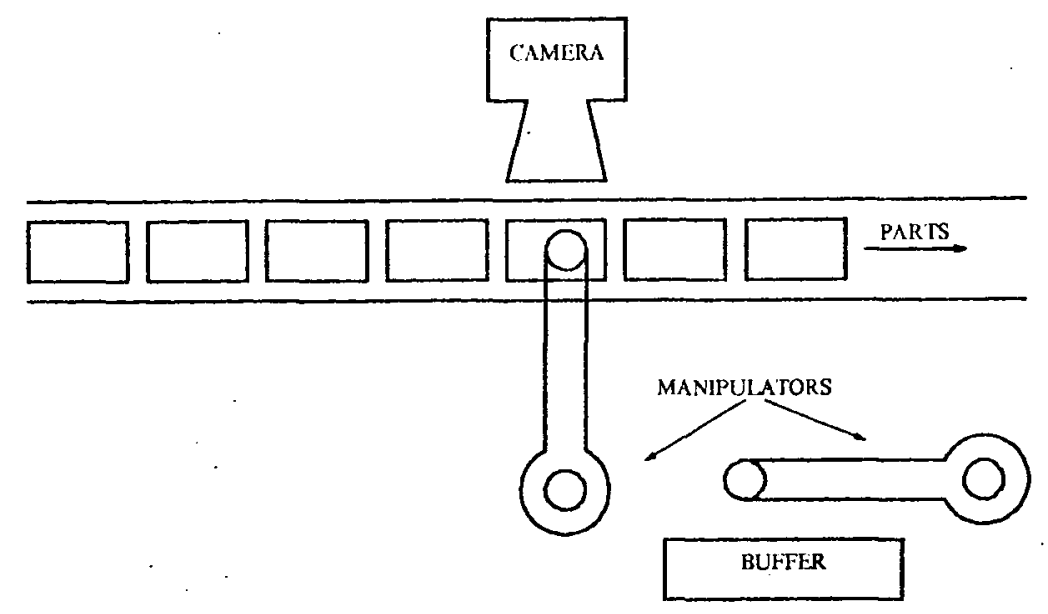

Figure 13: Robotic workstation

first precedence diagram of figure 3 (which combines A-B-C and B-A.C) as an example of precedence graph representation. Similar results will be produced using the other fixed sequences or precedence graphs. The number of operations that would be performed for each one of the 24 possible orderings in which the four parts of the simple product can be acquired is shown in Table 2. At least 7 operations are necessary: four acquisitions and three matings; depending on the order in which the parts are presented, buffering, and therefore retrieving may also be necessary.

When using the fixed sequence representation of plans, extensive buffering is necessary. For example, if the order the parts come is S HRC (stick, handle, receptacle, and cap) both the stick and the handle must be buffered since they are not used in the first operation; adding two bufferings and two retrievings to the four acquisitions and three matings that are always necessary yields 11 operations. The average number of operations for all 24 possiblc orders is 9.8 .

Using precedence diagrams for the representation of plans avoids some of the buffering and reduces the average number of operations to 9.2. For the sequence SHRC, for example, only the handle must be buffered since the insertion of the stick into the receptacle may be the first operation.

Using the AND-OR graph representation of plans, however, avoids most of the buffering, and yields the average of 8 operations. For the same SHRC sequence, for example, no buffering is needed because the robot can follow the sequence of operations corresponding to the solution tree shown in figure 6. 
Table 2: Number of operations necded to asscmble the product of fig. 1 for all the sequences in which the parts may be acquired, and for the three schemes of plan representation.

$$
\mathrm{C}=\text { cap } \mathrm{S}=\text { stick } \mathrm{R}=\text { receptacle } \mathrm{H}=\text { handle }
$$

\begin{tabular}{|c|c|c|c|}
\hline scquence & $\begin{array}{l}\text { first sequence } \\
\text { of figure } 2\end{array}$ & $\begin{array}{l}\text { first precedence diagrams } \\
\text { of figure } 3\end{array}$ & $\begin{array}{l}\text { AND/OR graph } \\
\text { of figure } 4\end{array}$ \\
\hline CSRH & 9 & 9 & 7 \\
\hline CSHR & 11 & 11 & 9 \\
\hline CRSH & 7 & 7 & 7 \\
\hline CRHS & 9 & 9 & 9 \\
\hline CHSR & 11 & 11 & 9 \\
\hline CHRS & 9 & 9 & 9 \\
\hline$S C R H$ & 9 & 9 & 7 \\
\hline $\mathrm{SCHR}$ & 11 & 11 & 9 \\
\hline $\mathrm{SRCH}$ & 9 & 7 & 7 \\
\hline SRHC & 11 & 9 & 7 \\
\hline SHCR & 11 & 11 & 9 \\
\hline S H R C & 11 & 9 & 7 \\
\hline $\mathrm{RCSH}$ & 7 & 7 & 7 \\
\hline $\mathrm{RCHS}$ & 9 & 9 & 9 \\
\hline $\mathrm{RSCH}$ & 9 & 7 & 7 \\
\hline RSHC & 11 & 9 & 7 \\
\hline RHCS & 9 & 9 & 9 \\
\hline R H S C & 11 & 9 & 7 \\
\hline HCSR & 11 & 11 & 9 \\
\hline HCRS & 9 & 9 & 9 \\
\hline H S C R & 11 & 11 & 9 \\
\hline H S R C & 11 & 9 & 7 \\
\hline H R CS & 9 & 9 & 9 \\
\hline H R SC & 11 & 9 & 7 \\
\hline average & 9.8 & 9.2 & 8 \\
\hline
\end{tabular}




\section{Conclusion}

$\Lambda$ compact representation for the set of all possible assembly plans of a product has been presented, along with its applications in the selection of the best assembly plan and in opportunistic scheduling. One important feature of that representation is that it allows one to traverse the space of all possible assembly plans, and therefore provides an opportunity to select an optimal schedule and dynamically adapt scheduling to changing conditions. Both the fixed sequence representation and the precedence diagram representation are very limited in this aspect.

$\Lambda$ number of issues related to this representation are under investigation. One important issue is the devclopment of algorithms for opportunistic scheduling suitable for real time operation. As pointed out in section 7, some buffering could not be avoided, even with the use of AND/OR graph representation of plans. For complex products, the choice of which part or subassembly to buffer may affect the overall assembly efficiency and criteria for that decision will be necessary. These criteria will certainly depend on evaluation functions, also under investigation, used to sclect a plan, cspecially functions that do not possess the recursive property like the one used in section 6 .

An additional important ongoing research issue is the development of a representation of assemblies suitable for the automatic generation of plans. Such automation can be helpful in design of both new products and assembly systems. In designing new products, the designer can quickly assess the difficulty of assembling and eventually modify the design to facilitate the assembly. In designing new assembly systems, the designer can evaluate the performance of a proposed design for a given set of products. 


\section{References}

[1] Bcllman, R. et al.

Mathematical Aspects of Scheduling and Applications.

Pergamon Press, 1982.

[2] Fahlman, Scott Elliott.

A Planning System for Robot Construction Tasks.

Artificial Intelligence 5(1):1-49, 1974.

[3] Fikes, Richard E. and Nilsson, Nils J.

STRIPS: $\Lambda$ New Approach to the Application of Theorem Proving to Problem Solving. Artificial Intelligence 2:189-208, 1971.

[4] Fikes, Richard E. et al.

Learning and Executing Generalized Robot Plans.

Artificial Intelligence 3:251-288, 1972.

[5] Fox, B. R. and Kempf, K. G.

Opportunistic Scheduling for Robotics Assembly.

In 1985 IEEE International Conference on Robotics and Automation, pages 880-889. IEEE Computer

Society, 1985.

[6] Fox, Mark S.

Constraint-Directed Search: A Case Study of Job-Shop Scheduling.

$\mathrm{PhD}$ thesis, Carnegie-Mellon University, december, 1983.

Also published as technical reports CMU-CS-83-161 and CMU-RI-TR-83-22.

[7] Krogh, Bruce H. and Sanderson, Arthur C.

Modeling and Control of Assembly Tasks and Systems.

Technical Report CMU-RI-TR-86-1, Robotics Institute - Carnegie-Mellon University, 1985.

[8] Nilsson, Nils J.

Principles of Artificial Intelligence.

Springer-Verlag, 1980.

[9] Sacerdoti, Earl D.

A Structure for Plans and Behavior.

Elsevier North-Holland, 1977. 